Check for updates

Cite this: Mater. Chem. Front., 2020, 4, 3153

Received 22nd July 2020, Accepted 14th August 2020

DOI: 10.1039/d0qm00522c

rsc.li/frontiers-materials

\section{Fusion of aggregation-induced emission and photochromics for promising photoresponsive smart materials}

\author{
Qing Yan (D) * and Sheng Wang (D) *
}

\begin{abstract}
Photochromic materials have been drawing considerable attention owing to their potential applications including optical devices, logic gates, data recording and storage, and chemosensors. However, most conventional photochromic compounds usually suffer from aggregation-caused quenching (ACQ), and the solid or aggregate state is often required for practical applications. The introduction of the aggregation-induced emission (AIE) property into photochromic systems not only relieves the limitation posed by the ACQ phenomenon, but also endows photochromic systems with more fascinating fluorescence properties. Therefore, novel classes of AlE-active photochromic materials have been reported and considerable efforts have been continuously devoted towards improving their functions and performances for matching the requirements of practical applications. This review systematically describes recent progresses made in the AIE-active photochromic materials with respect to molecular structures, material morphologies, and practical applications, while the principle of molecular design and mechanism of performance are also covered. Moreover, the challenges, emerging fields, development tendency, and some perspectives for AIE-active photochromic materials are discussed and presented.
\end{abstract}

School of Chemistry and Chemical Engineering, Key Laboratory of Clean Energy Materials Chemistry of Guangdong Higher Education Institutes, Lingnan Normal University, Zhanjiang, 524048, China. E-mail: dyesws@126.com;

Fax: +86-759-318-2228; Tel: +86-759-318-2515

\section{Introduction}

The world is beautiful and variable because of plentiful colors. Colors are not only aesthetic and a visual delight, but also function as an information signal. As a curious animal, chameleons exhibit reversible changes in skin colors depending on changes in the environment, such as courtship and camouflage. ${ }^{1}$

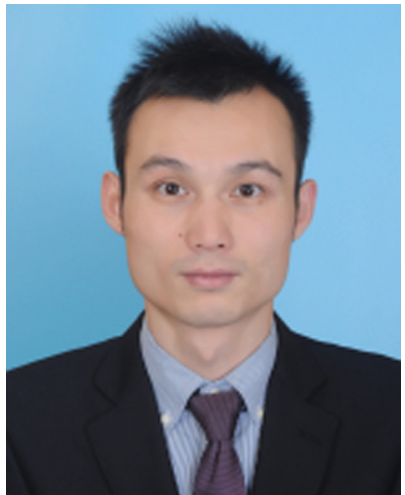

Qing Yan
Qing Yan was born in 1988. He received his BS in 2011 and master's degree in 2014 from Xiangtan University. He then, pursued his PhD in Tokyo Metropolitan University under the supervision of Prof. Nomura and obtained the degree in 2017. From January 2018, he worked in Lingnan Normal University as a Lecturer. His current research interests are focused on functional photochromic materials, organic dyes, and AIE materials.

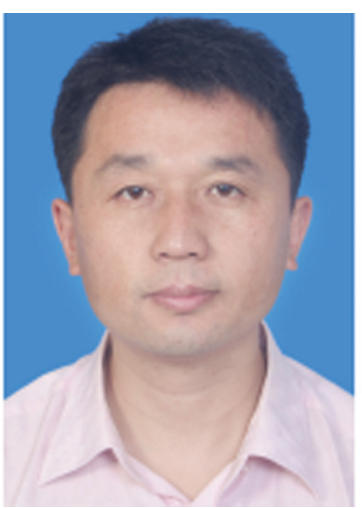

Sheng Wang
Sheng Wang received his PhD from ECUST in 2006 under the supervision of Prof. He Tian. From 2006 to 2008, he worked as a Postdoctoral under the supervision of Prof. Sung-hoon Kim at Kyungpook National University. From November 2010 to March 2011, he stayed in Prof. Young-A Son's lab at Chungnam National University as a Visiting Professor. From 2012 to 2013, he worked as a Postdoctoral Associate at Kent State University.

His current research mainly focuses on organic photochromic materials and organic functional dyes. Now, he works as a Full-time Professor at Lingnan Normal University. 
Learning from nature is still a hot topic and an effective strategy for developing new concepts and practical inventions, and tremendous achievements had been made and emulated by humans. Similar to the chameleon's skin, photochromic materials are smart materials that can reversibly change color while accompanied by various other changes in their photochemical and photophysical properties under light stimuli. ${ }^{2}$ Reversibility and driving force (light stimuli) are outstanding properties and advantages of photochromic materials compared with other stimuli-response smart materials ${ }^{3-6}$ (mechanical force, ${ }^{4} \mathrm{pH}^{5}$ or thermal energy ${ }^{6}$ ). Therefore, there has been an increasing interest in this domain ${ }^{7}$ and a tremendous number of publications have been reported in many fields, such as photoresponsive materials, ${ }^{8}$ biochemistry, ${ }^{9}$ supramolecular chemistry, ${ }^{10}$ pharmaceuticals, ${ }^{11}$ and catalytic reactions. ${ }^{12}$ Until now, most studies on organic photochromism have mainly focused on several families of compounds: azobenzenes/stilbenes, ${ }^{13}$ diarylethenes (DAE), ${ }^{14}$ spiropyrans (SP)/spirooxazines (SO) ${ }^{15}$ salicylideneaniline, ${ }^{16}$ and donor-acceptor Stenhouse adducts (DASAs). ${ }^{17}$ However, rational molecular design and precise molecular engineering can endow limited compounds with infinite possibilities. Each family of photochromic molecules has its specific properties, which can also be tuned via the functional modification of molecular structures. In short, a marginal geometrical or electronic modification can generate big differences in properties, and lot of efforts have been made to tune these molecules. ${ }^{18}$

Besides, to develop photochromic compounds with the desired functions for practical applications, considerable attention has been focused on designing or tuning photochromic systems with better fundamental properties such as higher quantum yield, good thermal stability, faster switching speed, and excellent fatigue resistance. More importantly, the solid or aggregate state is often required for practical applications, and the molecular structure also shows significant influence on its performance in the solid or aggregate state. Tuning the properties of photochromic molecules needs a thorough understanding of the relationships between structures and performances. However, most conventional systems based on photochromic compounds usually suffer from aggregation-caused quenching (ACQ). Therefore, addressing the limitations posed by this ACQ phenomenon has been the grand challenge in this research area. Significantly, the pioneering work involving aggregation-induced emission (AIE) was reported by Tang and coworkers in $2001,{ }^{19}$ which provided a powerful solution for the ACQ problem and offered a novel strategy to fabricate practical, organic luminescent materials with intense fluorescence in their aggregated or solid states. Since then, AIE systems have received increasing attention and have seen tremendous expansion in several fields such as organic light-emitting diodes (OLEDs), chemosensors, and other functional luminescent materials. ${ }^{20}$

In addition, AIEgens have also been applied as photochromic materials, and a variety of photochromic compounds exhibiting the AIE feature have been reported, too. ${ }^{21}$ Further, AIE has been considered to be an efficient solution for resolving the limitation posed by the ACQ problem in photochromic compounds.
Generally, the introduction of AIE into a photochromic system can be summarized into four paths: (1) direct mixing of AIEgens with photochromic molecules, (2) combination between AIEgens and photochromic molecules via chemical bonding, (3) combination between AIEgens and photochromic molecules via hostguest interactions, and (4) novel molecular system design. To the best of our knowledge, this is the first review that systematically summarizes the development of AIE-active photochromic materials. In this review, the advancements and emerging fields with regard to AIE-active photochromic materials are summarized and highlighted. They are classified as per specific molecular structures, material morphologies, and practical applications (Fig. 1), while the design principle and mechanism are also covered. This review aims to inspire more researchers to investigate the marriage between AIE and photochromism for producing more promising smart, photoresponsive materials.

\section{AIE-active photochromic molecules}

\subsection{AIE-active DAE}

DAE-one of the most popular kind of photochromic molecules-is a thermally irreversible photochromic switch. It can undergo a reversible photocyclization reaction between the unconjugated ring-opening isomer and conjugated ringclosing isomer via the irradiation of appropriate light sources.

A combination of AIEgens with photochromic molecules is the simplest and most efficient strategy to induce the AIE property into photochromic molecules. ${ }^{21}$ Recently, Wang et al. reported a series of novel DAE derivatives 1a-9a possessing both AIE (or aggregation-induced enhanced emission, AIEE) and photochromism properties by conjugating tetraphenylethene (TPE) or triphenylethene (TrPE) moieties into bisthienylethene (BTE), as shown in Fig. 2. ${ }^{2,23}$ All these compounds exhibited good photochromic properties both in the solution and solid states when irradiated with alternating UV and visible light. As expected, all of them exhibited typical AIE or AIEE behavior in a THF/water mixed solution, almost no fluorescence in a pure THF solution, but strong emission in their solid or aggregate states. Moreover, the photochromic and fluorescence properties could be tuned by the side substituents. Compounds (4a, 7a) connecting TrPE to BTE exhibited weaker fluorescence emission than the ones consisting of TPE and BTE in their solid or aggregate states. It is noteworthy that a traditional ACQ luminophore (triphenylamine) could exhibit the AIE property by introducing the AIE unit (TPE or TrPE) into the ACQ molecules, which provided a novel strategy toward the design of AIE-active photochromic DAE systems. Furthermore, rewritable information storage could be successfully achieved by a polymethyl methacrylate (PMMA) film.

Except for the above TPE-based DAE molecules, Zhu and coworkers reported a novel AIE-active photochromic DAE molecule (10a) based on the quinoline-malononitrile (EQ) fluorophore. ${ }^{24} \mathbf{1 0 a}$ afforded a strong emission at $613 \mathrm{~nm}$ in the aggregation state formed by increasing the water fraction $\left(f_{\mathrm{w}}\right)$ of its $\mathrm{THF} / \mathrm{H}_{2} \mathrm{O}$ mixed solution (Fig. 3A). Interestingly, the color of the solution or spectra 


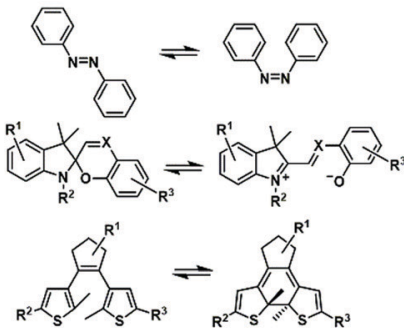

Photochromic molecules

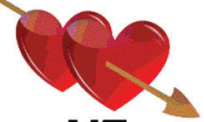
AIE

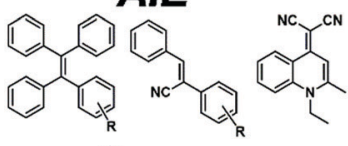<smiles>C1=CC(=C(c2ccccc2)c2ccccc2)C=C1</smiles>

Material Morphology

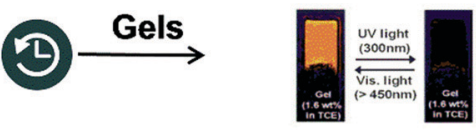
$\leftrightarrow \stackrel{\text { liquid crystals }}{\longrightarrow}$

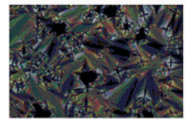

$A \stackrel{\text { Polyme }}{\longrightarrow}$ ㄷ. Supramolecules

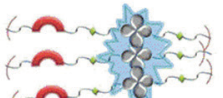
a<smiles>COC1CC(O)C(O)C(O)C(O)C(O)C1O</smiles>

\section{Nanoparticle}
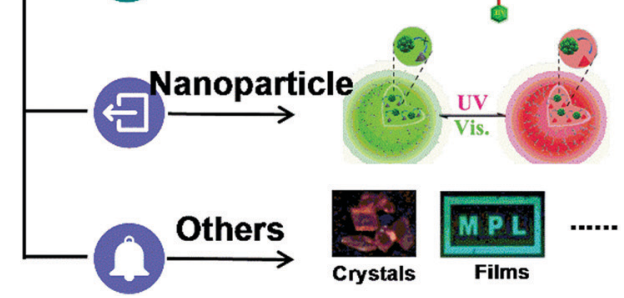

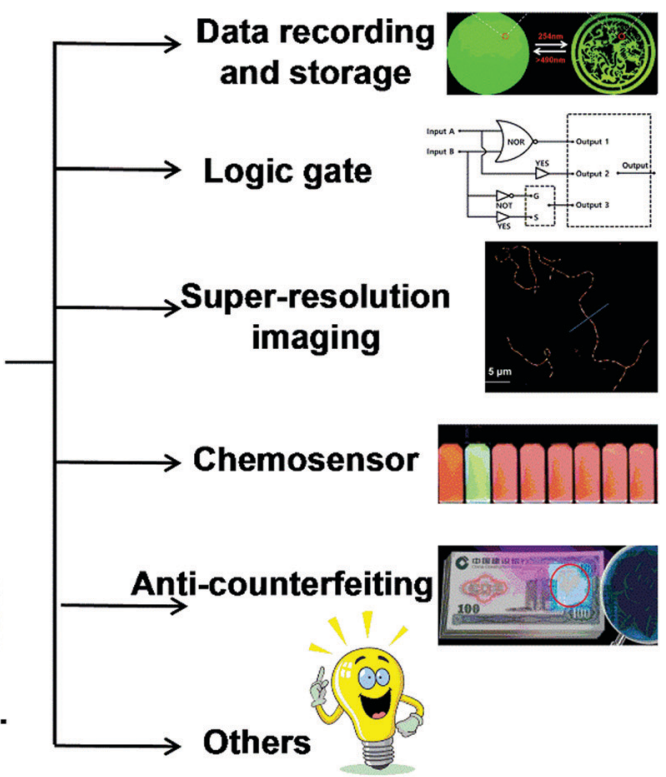

Fig. 1 Illustration of the material morphology and applications of AIE-active photochromic molecules. Material morphology from up to down: reproduced with permission. ${ }^{51}$ Copyright 2009, Wiley-VCH. Reproduced with permission. ${ }^{58}$ Copyright 2018, Royal Society of Chemistry. Reproduced with permission. ${ }^{61}$ Copyright 2017, Royal Society of Chemistry. Reproduced with permission. ${ }^{77}$ Copyright 2019, American Chemical Society. Reproduced with permission. ${ }^{72}$ Copyright 2017, Royal Society of Chemistry. Reproduced with permission. ${ }^{46}$ Copyright 2016, Royal Society of Chemistry. Reproduced with permission. ${ }^{59}$ Copyright 2005, American Chemical Society. Applications from up to down: reproduced with permission. ${ }^{81}$ Copyright 2018, American Chemical Society. Reproduced with permission. ${ }^{52}$ Copyright 2018, Wiley-VCH. Reproduced with permission. ${ }^{85}$ Copyright 2019 , Wiley-VCH. Reproduced with permission. ${ }^{65}$ Copyright 2019, American Chemical Society. Reproduced with permission. ${ }^{49}$ Copyright 2019 , Wiley-VCH.

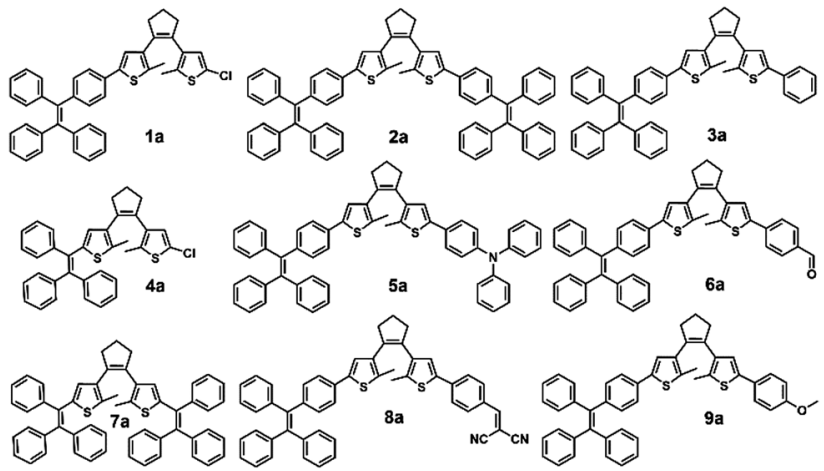

Fig. 2 Structures of AlE-active DAE derivatives 1a-9a.

did not change upon $365 \mathrm{~nm}$ UV-light irradiation when the $f_{\mathrm{w}}$ value reached $90 \%$, indicating the inactivation of photochromism. This unexpected aggregation-blocked photocyclization could be explained by the formation of the possible AIE state of 10c. However, the photochromic activity could be fully recovered by decreasing the $f_{\mathrm{w}}$ value to $50 \%$ by adding THF. Uchida et al. synthesized the new DAE derivative of 11a containing the excitedstate intramolecular proton transfer (ESIPT) functional moiety, as shown in Fig. 3B. ${ }^{25}$ The fluorescence of 11a was quenched by the increasing content of ring-closed isomers (11b) under UV-light irradiation in an aprotic solvent and in crystals, indicating
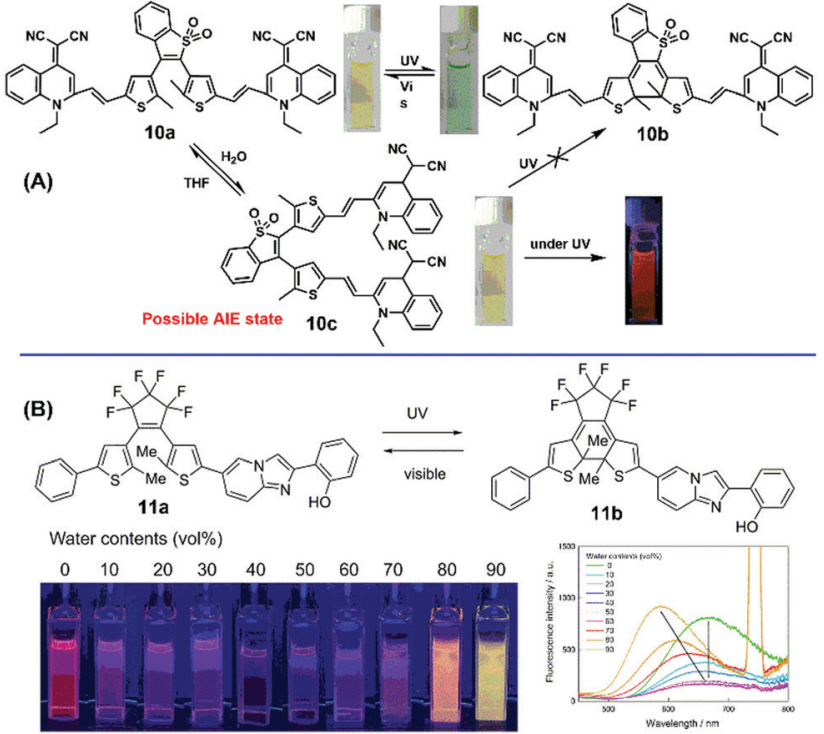

Fig. 3 (A) UV and visible light photochemically and reversibly converts 10a (ring-opened isomer) and 10b (ring-closed isomer). Reproduced with permission. ${ }^{24}$ Copyright 2017, Royal Society of Chemistry. (B) Photoisomerization of compound 11 and fluorescence properties of its solutions/ suspensions $\left(1.2 \times 10^{-4} \mathrm{M}\right)$ in THF/water mixtures with different water contents under UV-light irradiation. Reproduced with permission. ${ }^{25}$ Copyright 2019, Beilstein-Institut. 
turn-off-type fluorescence switching. In addition, protic and polar solvents also played a role in suppressing the fluorescence due to the inhibition of the ESIPT process. In the THF/ water mixed solution, the fluorescence of 11a was gradually quenched with an increase in the $f_{\mathrm{w}}$ value from 10 to $60 \mathrm{vol} \%$. Because of the AIE effect, a further increase in the $f_{\mathrm{w}}$ value led to a blue-shift in the emission band in addition to intense fluorescence. This system may have potential applications as sensors and color indicators.

Normally, it is worthwhile to combine different functionalities with various chemical or physical modifications when designing a multifunctional molecule. This strategy often works, but interference among the different units can occur and the synthesis routes as well as molecular structures often become complicated. Constructing multifunctional molecules via a simple synthesis route is fairly glamorous. Starting with commercially available reagents, Qu et al. developed simple hybrid tetraarylethenes 12a and 12c that comprised two benzenes and two aromatic thiophenes, as shown in Fig. $4 .^{26}$ As a result, the reversible photochromism and switchable fluorescence of trans-isomer 12a and cis-isomer 12c were observed in solution, solid powder, single crystal, and PMMA film. More interestingly, their AIE or ACQ behavior could be triggered by photoinduced isomeric conversion. Therefore, these versatile hybrid tetraarylethenes can be promising candidates for applications in logic gates, nondestructive readouts, and fluorescent photoswitches.

\subsection{AIE-active SP/SO}

$\mathrm{SP} / \mathrm{SO}$ are other types of well-known molecular switches that undergo ring-opening and ring-closing isomerization when irradiated with an appropriate light source. However, the weak emission in the aggregation state limits their applications. Therefore, the introduction of the AIE effect into the SP/SO molecules is a desirable strategy to improve their performances and expand their applicability.

$\mathrm{Xu}$ et al. reported two new SP molecules (13a and 14a) with the TPE moiety via carbon-carbon double-bond linkage, as shown in Fig. 5A. ${ }^{27}$ These two molecules exhibited AIE properties as well as photochromic and acidochromic properties.
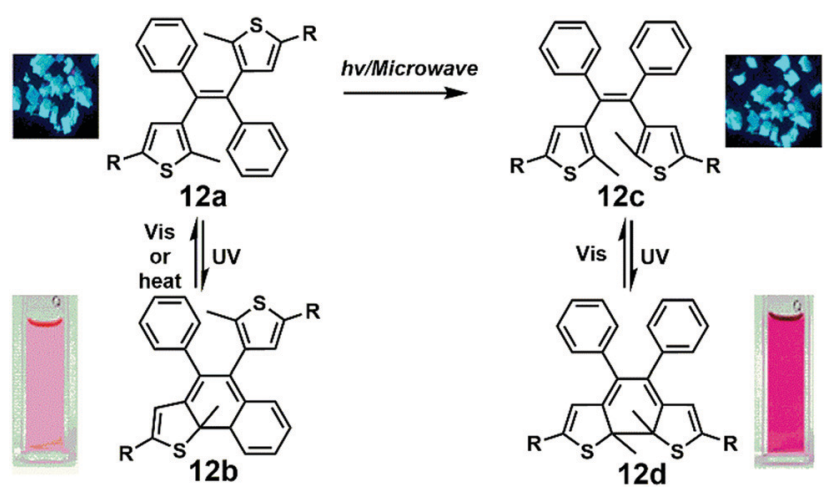

Fig. 4 Hybrid tetraarylethenes 12a with photoswitchable AIE and reversible photochromism. Reproduced with permission. ${ }^{26}$ Copyright 2017. American Chemical Society.
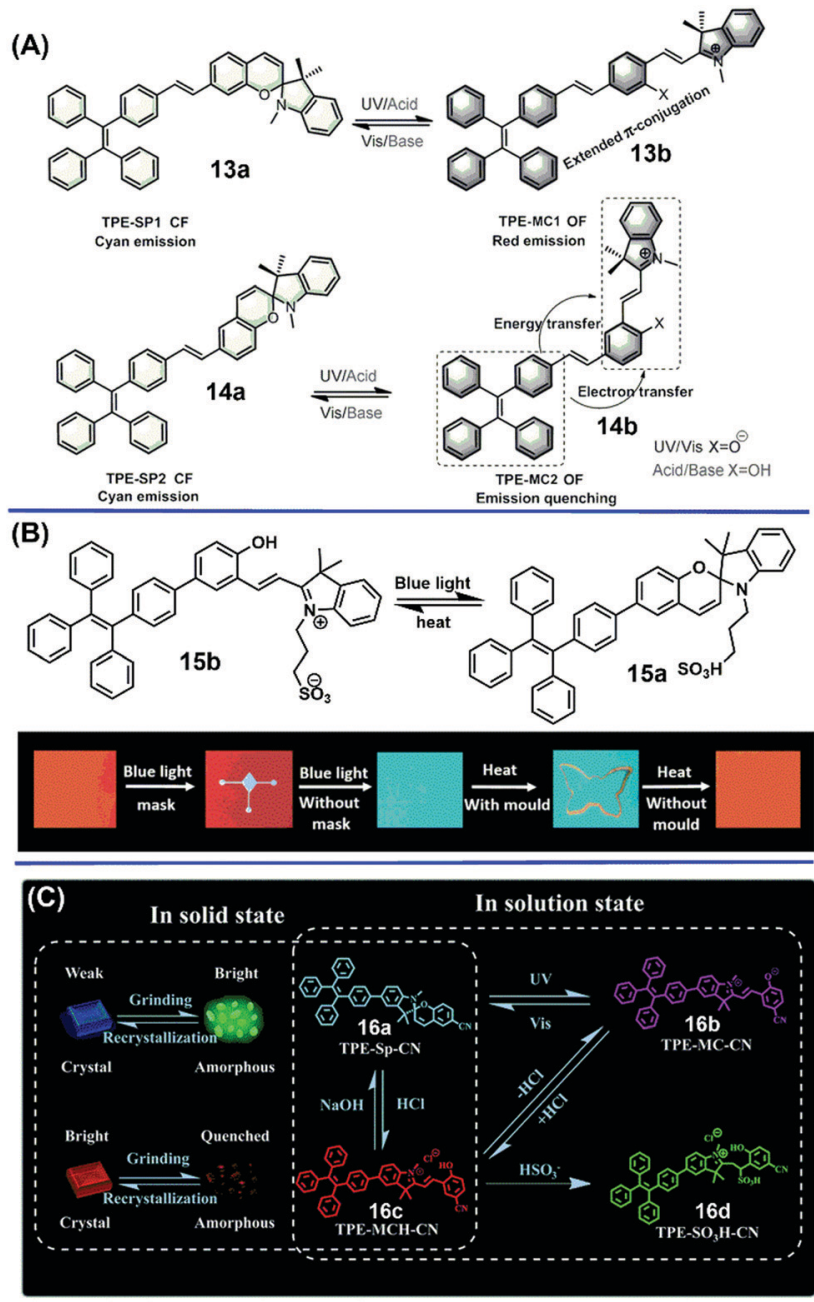

Fig. 5 (A) Possible origin for two different modes of fluorescent switches 13a and 14a. Reproduced with permission. ${ }^{27}$ Copyright 2015, Wiley-VCH. (B) Illustration of a rewritable display with a SPPATPE/PVP/PDMS hybrid film sequentially using blue light and heat as the writing and erasing stimuli. The size of the film was $2 \mathrm{~cm} \times 2 \mathrm{~cm}$ and the photographs were taken under $365 \mathrm{~nm}$ irradiation. Reproduced with permission. ${ }^{28}$ Copyright 2018, Royal Society of Chemistry. (C) Illustration of the switching states of 16a with different stimuli. Reproduced with permission. ${ }^{29}$ Copyright 2019, Royal Society of Chemistry.

Interestingly, 13a and 14a were similar structures but the linking positions between the TPE and SP were different, which resulted in different photochromic properties. 13a yielded red-emissive 13b owing to better conjugation upon UV-light irradiation. However, 14a generated emission quenching of open-form 14b owing to the combination of energy and electron-transfer processes. Later, Liu's group developed a novel multi-stimuli-responsive fluorescent switch (15b) via a covalent bond between SP photoacid and TPE molecule. ${ }^{28}$ The cyclization reaction of ring-opened $\mathbf{1 5 b}$ could be triggered by visible light or basic solution. Both opening and closing forms exhibited typical AIE properties: no fluorescence in the solution state, but strong fluorescence in the aggregation state. Furthermore, the hybrid polymer film of $\mathbf{1 5 b}$ was prepared, and the noninvasive stimuli of blue light and heating could be a writing or erasing tool, 
as shown in Fig. 5B. The original pattern was printed on the film of the ring-opened form by using a photomask under blue-light irradiation. If the photomask was removed, the entire film changed to a cyan emission upon further irradiation of blue light. Then, another pattern could be printed using a heated mold, and the film recovered to the initial state upon further heating. Liu et al. reported another TPE-modified cyano-SP (16a) with multi-stimuli-responsive properties, such as light, $\mathrm{pH}$, sulfite anion, and hydrostatic pressure. ${ }^{29}$ As shown in Fig. 5C, small-molecule 16a exhibited four fluorescent states both in the solution and solid states and they could reversibly convert to another under a suitable stimulus. Besides, the optical signals of $\mathbf{1 6 a}$ showed good linear relationship with external stimuli, which might be useful for multiparameter analysis in complex environments, logic gates, super-resolution imaging, and other fields.

Except for the above TPE-based SPs, Xu et al. were the first to synthesize 17a (Fig. 6a) by using another representative AIEgen of 9,10-distyrylanthrance (DSA) and two SP moieties via an esterification reaction. ${ }^{30}$ The twist conformation and considerable steric hindrance of DSA resulted in the loose packing of 17a in the solid state, which facilitated efficient tautomerization. Notably, under alternate UV-light and visible-light/heating treatments, highly reversible and efficient photoswitching could be obtained both in the solution and solid states. As shown in Fig. 6b, the yellow solid powder of 17a instantaneously turned into dark purple upon UV-light irradiation accompanied by a new emission band and absorption peak. This outstanding photochromic property of $\mathbf{1 7 a}$ in the solid state led to its successful implementation as a superresolution imaging agent and anticounterfeit ink.

Compared with SP, the AIE-active photochromic SO has been rarely reported. ${ }^{31,32}$ As an example, Yang's group ${ }^{31}$ synthesized TPE-SO (18a) by conjugating the photochromic SO group with a TPE fluorophore, as shown in Fig. 7. When the $f_{\mathrm{w}}$ value was higher than $70 \%$ in $\mathrm{THF} /$ water mixed solution of $18 \mathrm{a}$, the emission peak at $475 \mathrm{~nm}$ could be clearly observed in addition to the fluorescent images under UV illumination at $365 \mathrm{~nm}$, which demonstrated typical AIE behavior. In addition, reversible photochromism was also investigated.

\subsection{AIE-active azobenzenes}

Azobenzenes are typical photochromic molecules that undergo reversible $\mathrm{N}=\mathrm{N}$ trans-cis isomerization reactions upon $\mathrm{UV}$ - and

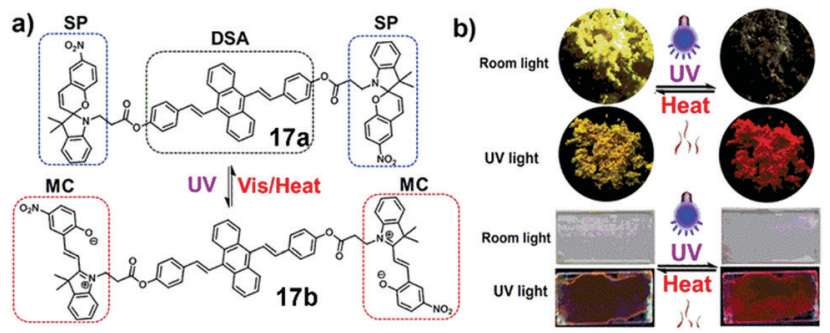

Fig. 6 (a) Reversible structural isomerization between 17a and 17b under different stimuli. (b) Visible and fluorescent photographs of the powders and films of 17a. Reproduced with permission. ${ }^{30}$ Copyright 2017, American Chemical Society.

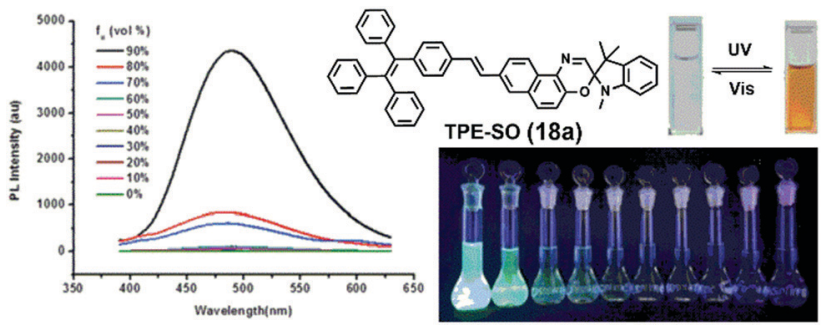

Fig. 7 Structure of $\mathbf{1 8 a}$ and its AIE and photochromic properties. Reproduced with permission. ${ }^{31}$ Copyright 2019, Royal Society of Chemistry.

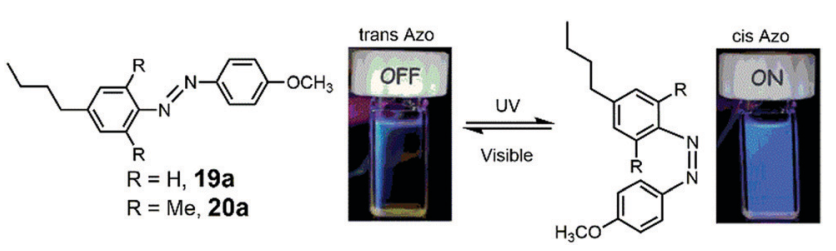

Fig. 8 Structures of $19 a$ and $20 a$, and visualized images of photoswitchable fluorescence "on/off" behavior of 19a. Reproduced with permission. ${ }^{34}$ Copyright 2012, Royal Society of Chemistry.

visible-light irradiation. ${ }^{33} \mathrm{Chu}$ et al. designed two novel azobenzene derivatives 19a and 20a, as shown in Fig. $8 .^{34}$ The blue fluorescence from both could be rapidly switched on upon UV-light irradiation, and the nonfluorescence of solution 19a was retained under the subsequent irradiation of visible light, while moderate emission was maintained for solution 20a. The authors proposed that the difference between these two azobenzenes in the photostationary state (PSS) of visible light could be attributed to the AIE effect of the bent-shaped cis form of two methyl-substituted 20a. This steady fluorescence of cis-20a could find applications in molecular sensing and bioimaging.

\subsection{AIE-active photochromic Schiff-base derivatives}

Compared with well-known photochromic molecules, few studies have investigated photochromic Schiff-base derivatives, such as hydrazone or salicylaldehyde derivatives. They could also exhibit excellent photochromic property via a rational molecular design. The mechanism of photochromism in these compounds has always been intertwined with the ESIPT process, as shown in Fig. 9. Moreover, the magical power of AIEgens can induce various possibilities to develop novel Schiff-base derivatives with excellent photochromic properties.

Wang et al. reported two novel Schiff-base derivatives $\mathbf{2 1}$ and 22 (Fig. 9) by connecting the TPE and cholesterol moieties into the salicylideneaniline group. ${ }^{35}$ Interestingly, molecule 22 had one more phenyl ring than 21, which resulted in different AIE, photochromism, and gelation properties. Gelation-induced enhanced emission was noted from the gel of molecule 22 formed in cyclohexane, and alternate cooling and heating could control the gel-solution transition as well as fluorescence intensity. Besides, the characteristic photochromic behaviors were demonstrated in the aggregation state (gel and solid) under UV-light irradiation. Li et al. developed the simple reversible 


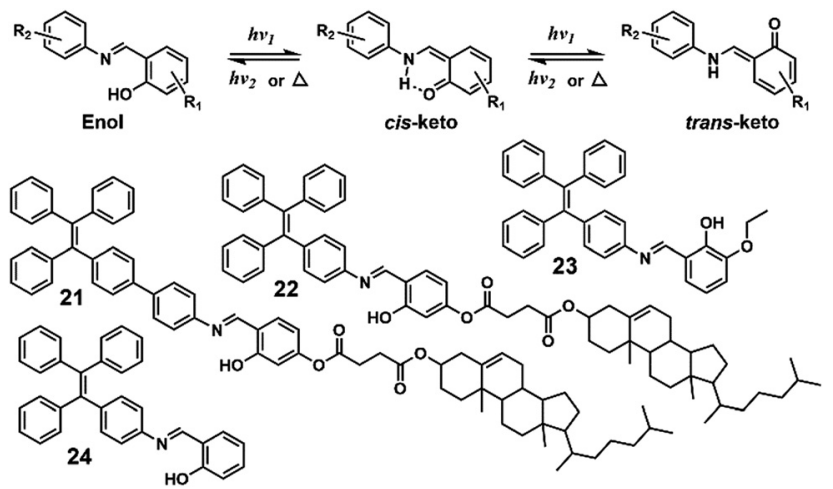

Fig. 9 Chemical structures of TPE-based Schiff-base derivatives 21-24 and their photochromic mechanisms.

photochromic AIE molecule of 23 by directly connecting salicylideneaniline with the TPE group. ${ }^{36}$ Typical AIE phenomenon could be observed in $\mathrm{H}_{2} \mathrm{O} / \mathrm{EtOH}$ mixtures as the $f_{\mathrm{w}}$ value increased. Switchable visual color and fluorescence could be triggered with good fatigue resistance via UV-light irradiation, and the manipulation of thermal- and long-wavelength-light irradiation could be used to control the recovery rate from trans-23-keto to 23-enol. Recently, Zhang et al. modified 23 with an additional ethoxy group to afford $24 .{ }^{37}$ Notably, 24 could rapidly switch on/off by using alternating $410 \mathrm{~nm}$-light and whitelight irradiation with excellent fatigue resistance. Moreover, grinding obviously enhanced the fluorescence intensity of $\mathbf{2 4}$ accompanied by lower photoresponse sensitivity and photochromic efficiency. Importantly, 24 could be successfully used as rewritable paper, which might prove to be useful in reducing paper production and consumption.

Moreover, Jia's group synthesized a series of pyrazolone phenylsemicarbazone derivatives 25a, as shown in Fig. 10A. ${ }^{38}$ They did not exhibit luminescence in pure ethanol; a higher $f_{\mathrm{w}}$ value resulted in higher luminescence intensity until the $f_{\mathrm{w}}$

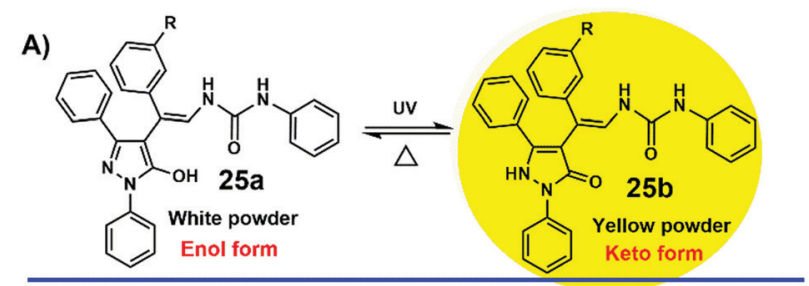

B)
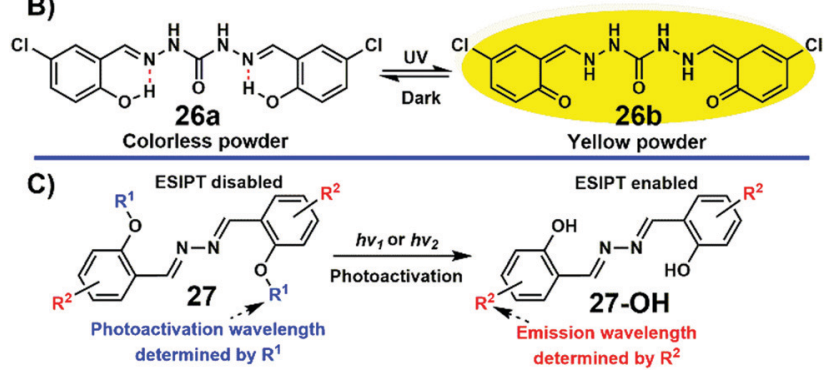

Fig. 10 Chemical structures of TPE-based Schiff-base derivatives 25-27 and their corresponding photochromic mechanisms. value reached $80 \%$, indicating typical AIE behavior. With an increase in aging time, 1D nanorods could form 0D nanoballs during aggregation. Moreover, these compounds possessed reversible photochromic properties in the solid state with excellent fatigue resistance upon UV-light irradiation or heating, while the color changed between white and yellow. Liu et al. reported simple Schiff-base 26a (Fig. 10B) that exhibited photo-controllable color changes in the solid state and AIE characteristics in an aqueous solution. ${ }^{39}$ The crystals of $26 \mathbf{a}$ could be transferred from colorless to yellow upon UV-light irradiation and recover to colorless again in the dark due to the reversible intramolecular proton transfer process. In addition, based on 26a and rhodamine B, highly efficient light-harvesting systems could be successfully fabricated in an aqueous solution, where 26a served as an energy donor and rhodamine $\mathrm{B}$ acted as an acceptor via the Förster resonance energy transfer (FRET) process. Notably, the addition of $\mathrm{Al}^{3+}$ or $\mathrm{Cu}^{2+}$ ions could destroy the $\mathrm{N} \cdots \mathrm{H}-\mathrm{O}$ hydrogen bond resulting in different emissions; this trait can be exploited in chemosensing toward the detection of $\mathrm{Al}^{3+}$ or $\mathrm{Cu}^{2+}$ ions. Therefore, such a multifunctional material possessing reversible photochromism, AIE activity, light-harvesting ability, and cation detection provided more possibilities for multifunctional molecular design.

As mentioned above, the ESIPT process played an important role in the photochromism of hydroxyl-containing compounds. However, Xiang et al. developed a series of caged salicylaldehyde hydrazone derivatives 27 in which the ESIPT process was disabled by the caging groups $\mathrm{R}^{1}$ (Fig. 10C). ${ }^{40}$ Interestingly, the photoactivation wavelength was determined by the caging group $\mathrm{R}^{1}$, and substitutions $\mathrm{R}^{2}$ on the salicylaldehyde hydrazone could affect the fluorescence emission. Therefore, ESIPT and intense fluorescence could recover in the aggregation state (colloidal solution and solid) under suitable light irradiation by removing the caging group $\mathrm{R}^{1}$ on the hydroxyl moiety. Moreover, these caged salicylaldehyde hydrazones were successfully utilized into multicolor photopatterning and photoactivatable cell imaging applications, which demonstrated their potential applicability in chemistry and biology as promising candidates for photoactivatable solid-state fluorophores.

\subsection{AIE-active DASAs}

DASAs, reported for the first time by J. Read de Alaniz's group in 2014, have attracted increasing attention in recent years. ${ }^{17,41}$ This new type of photochromic molecule can transfer from a strong colored triene form to a colorless cyclopentenone upon visible-light irradiation. Compared with traditional photochromic molecules, DASAs can prevent getting damaged from high-energy UV light, which is a promising requirement of biomaterials. He's group reported the first combination of TPE moiety with DASAs, as shown in Fig. 11A. ${ }^{41 a}$ This visiblelight-triggered AIE molecule (28a) exhibited outstanding photochromic properties with color changes between purple and colorless. Reversible changes in the absorption band and fluorescence intensity could be observed under alternate visible-light irradiation and heating, which could be attributed to the stimuli-responsive tautomerization between the triene 


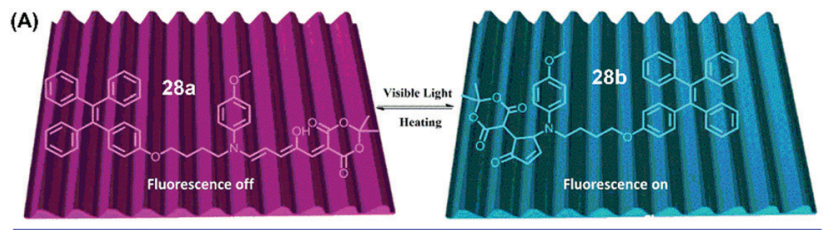

(B)

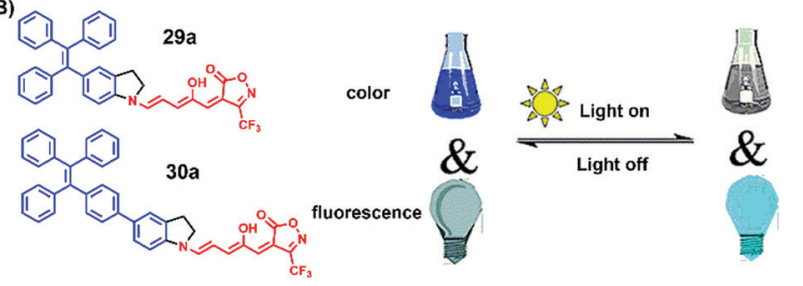

Fig. 11 (A) Photochromic property of 28a. Reproduced with permission. ${ }^{41 a}$ Copyright 2018, Royal Society of Chemistry. (B) Photochromic and fluorescence properties of 29a and 30a. Reproduced with permission. ${ }^{41 b}$ Copyright 2020, Elsevier

isomer and cyclopentenone isomer. The quenching mechanism for nonfluorescent triene isomers can be ascribed to the FRET from the TPE donor to the DASA acceptor. Moreover, visiblelight-triggered fluorescent surface relief patterns were fabricated and the subsequently formed PMMA films were also highlighted in this work. Recently, Wang's group synthesized two new AIEactive photochromic molecules (29a and 30a) containing thirdgeneration DASAs and TPE derivatives. ${ }^{41 b}$ As shown in Fig. 11B, the initial blue color converted to colorless upon visible-light irradiation in solution or film due to the formation of the cyclopentene isomer, while the fluorescence was switched on. Subsequent heating restored it to its original state. These three novel AIE-active photochromic molecules provided innovative strategies for developing visible-light-triggered fluorescent materials.

\subsection{Other AIE-active photochromic molecules}

Except for the typical photochromic molecules mentioned above, some other photochromic systems with AIE characteristics have been investigated. Tang's group developed simple cyanostilbene-based AIE-active fluorophore 31a. ${ }^{42}$ This molecule could undergo $Z / E$ tautomerization, photodimerization, and photocyclization under suitable conditions, such as aggregate morphology and irradiation light wavelength, as shown in Fig. 12A. Reversible $Z / E$ tautomerization occurred upon roomlight irradiation and heating in solution. Subsequent UV-light irradiation could result in a cyclization reaction accompanied by a dramatic enhancement in fluorescence, while photodimerization would take place via recrystallization. The detailed mechanism for these photoreactions was evidenced by experimental analyses and DFT calculations. It is worth noting that this kind of single AIEgen system with controllable multiple switches can provide a novel strategy to fabricate advanced multifunctional materials. Ling et al. reported symmetrically substituted diarylmaleic anhydrides 32a with the AIE property, as shown in Fig. 12B. ${ }^{43}$ This compound exhibited interesting photochromic phenomenon only in a DCM solution under UV-light irradiation. On prolonging the irradiation time, the colorless solution

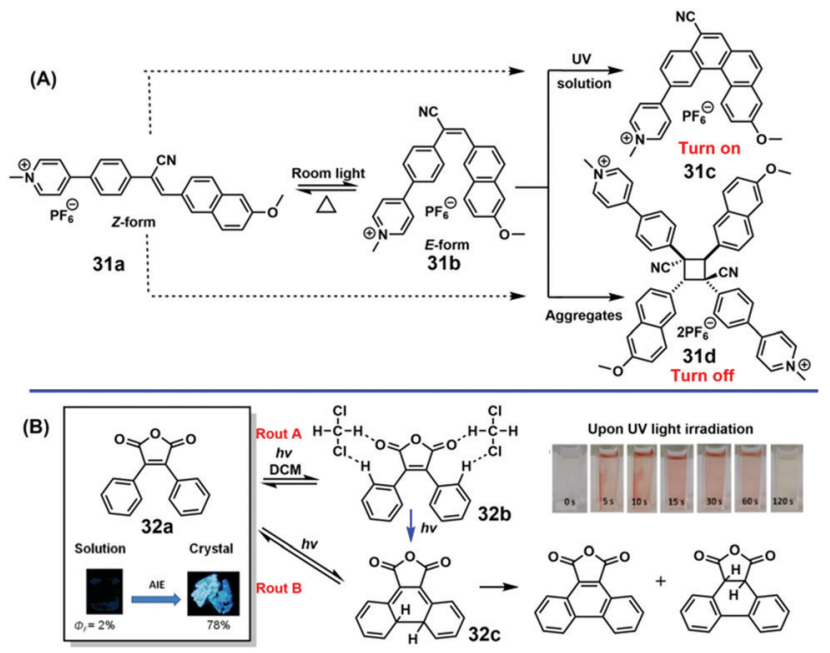

Fig. 12 (A) Chemical structures of $\mathbf{3 1 a}-\mathbf{d}$ and their corresponding photochromic mechanisms. (B) Chemical structures of $32 \mathrm{a}-\mathrm{c}$ and their AIE and photochromic properties. Reproduced with permission. ${ }^{43}$ Copyright 2017, Royal Society of Chemistry.

gradually turned into red and faded back to colorless after $120 \mathrm{~s}$ of irradiation. This behavior could be attributed to the existence of two reaction routes (A and $\mathrm{B}$ ) that were concomitant and competitive in the photochromism process in the DCM solution. Moreover, the introduction of additional substituents on this diarylmaleic anhydride could afford a series of full-color emissive derivatives, which offered a path for a dualstate emissive molecular design.

Ma et al. synthesized new multifunctional multi-stimuliresponsive molecule 33a by fusing rhodamine with TPE (Fig. 13A). ${ }^{44}$ This molecule exhibited AIE characteristics, methanol response, reversible photochromism, and mechanochromism. It was notable that 33a possessed three well-distinguished high contrast states of cyan, red, and dark in the solid state. The reversible switching between any two states could be controlled by the stimuli of UV/visible light, acid/base, or mechanical force/ chemical vapor, which would prove to be a new strategy for the development of multi-stimuli-responsive fluorescent switches. Yang et al. synthesized photochromic TPE-NP molecule 34a by conjugating the TPE moiety with the naphthopyran group. ${ }^{31}$

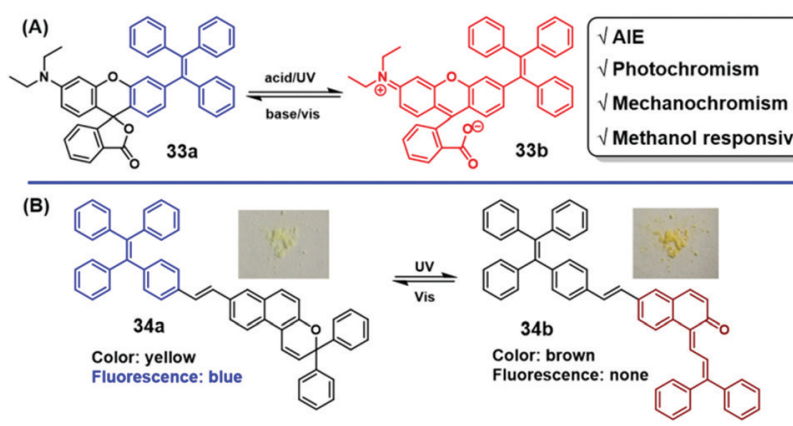

Fig. 13 (A) Chemical structures of $33 a-b$ and their corresponding photochromic reactions. (B) Chemical structures of $34 a-b$ and their corresponding photochromic reactions. 
This molecule showed the AIE phenomenon in $\mathrm{THF} / \mathrm{H}_{2} \mathrm{O}$ mixtures and reversible photochromism both in the solution and solid powder states, as shown in Fig. 13B. Particularly for the solid state, 34a showed color changes from yellow to brown upon UV-light irradiation, while the fluorescence was switched off due to the formation of open-form 34b. Moreover, this can be successfully applied as an anticounterfeiting ink.

Tang et al. reported a novel polycyclic hydrocarbon molecule with unexpected photochromic properties. ${ }^{45 a}$ As shown in Fig. 14A, colorless crystals of 35a changed to red and its blue emission was turned off under UV-light irradiation; the subsequent treatment under room light gradually recovered it to its initial state due to the ring-closing and ring-opening reactions. However, the photochromic process was not observed in solution, which indicated the aggregation-promoted photochromism phenomenon. Moreover, 35b could be irreversibly oxidized to obtain ACQ molecule 35c, which was the opposite of its precursor 35a. Subsequently, they synthesized a class of novel deep-blue materials fabricated by fusing flexible diphenylethene and a rigid spiro scaffold, as shown in Fig. 14B. ${ }^{45 b}$ 36b exhibited the typical AIE phenomenon with solid-state fluorescence quantum yield of up to $99.8 \%$.

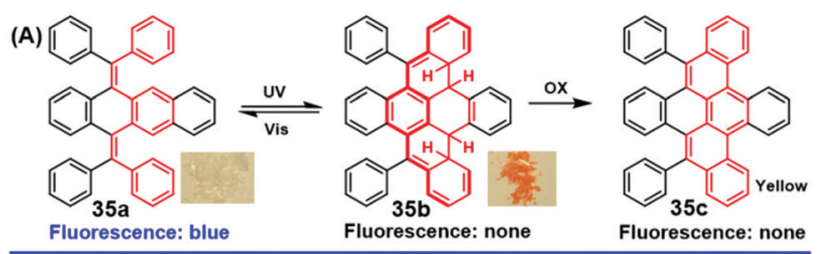

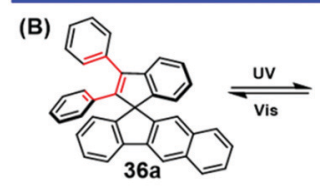

Deep blue emission Solid-state of up to $99.8 \%$

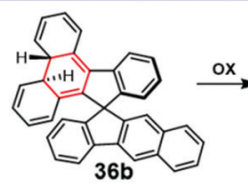

Photochromism Solution $\phi<-9 \%$

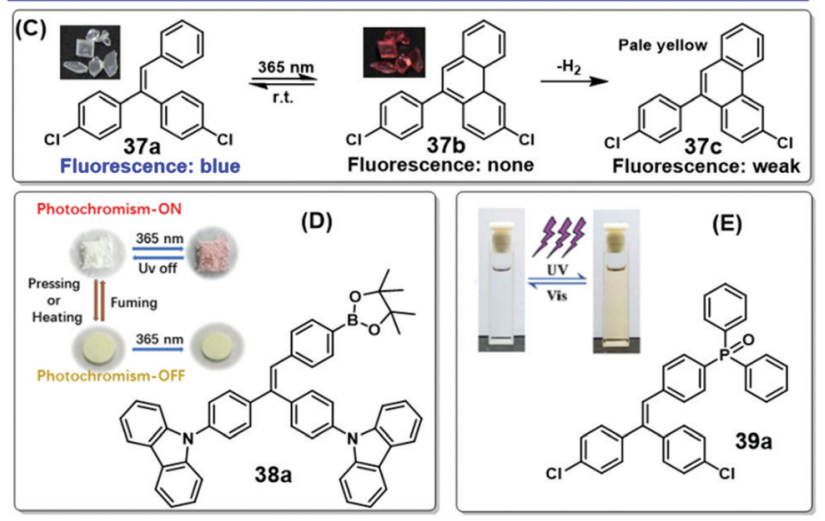

Fig. 14 (A) Chemical structures of $35 \mathrm{a}-\mathrm{c}$ and their photochromic reactions. (B) Chemical structures of $36 a-c$ and their photochromic reactions. (C) Chemical structures of $\mathbf{3 7} \mathbf{a}-\mathbf{b}$ and their photochromic reactions. Reproduced with permission. ${ }^{46}$ Copyright 2016, Royal Society of Chemistry. (D) Chemical structure of $\mathbf{3 8 a}$ and its photochromic reaction in the solid state. Reproduced with permission. ${ }^{47}$ Copyright 2017, Royal Society of Chemistry. (E) Chemical structure of $39 a$ and its photochromic reaction in solution. Reproduced with permission. ${ }^{48}$ Copyright 2018, Royal Society of Chemistry.
The reversible photocyclization on diphenylethene was identified in dilute solutions, but it was suppressed by aggregation, indicating an opposite behavior compared with that of $\mathbf{3 5}$. These two works demonstrated that photocyclization was a key process behind the restriction of intramolecular motion (RIM) mechanism. Chi's group developed a series of TrPE derivatives 37-39 with AIE and photochromism properties. ${ }^{46-48}$ As shown in Fig. 14C, colorless solid 37a rapidly changed to red and its blue fluorescence was subsequently switched off upon UV-light irradiation. The photochromic bleaching process also occurred after several seconds at ambient temperature, with the color recovering to colorless due to the fast ring-opening reactions. After irradiation for many cycles in air, pale-yellow-colored solid $37 \mathbf{c}$ was obtained by the dehydrogenation process of the closed form. ${ }^{46}$ In the case of 38a, the AIE, piezochromic, and photochromic properties were successfully demonstrated. ${ }^{47}$ Photochromism could only be observed in the crystalline state, and pressing/fuming or heating/fuming could switch on/off the photochromic properties, as shown in Fig. 14D. Different from the above TrPE derivatives, 39a (Fig. 14E) only exhibited distinct photochromic properties in solution, which could be attributed to the restriction of the ring-closing reaction by the sterichindered triphenylphosphine oxide moieties in the aggregation states. ${ }^{48}$ On the basis of the abovementioned efforts, a simple modification by different substituents would endow these compounds with significant differences in their photochromic properties and functions.

Recently, Li and Tang's group synthesized $\mathbf{4 0}$ by a one-step reaction between a TPE moiety and cholesterol moiety. ${ }^{49}$ This novel TPE derivative exhibited AIE, self-recovery photochromism, and multistate mechanochromism. As shown in Fig. 15, white powder 1p-h or transparent crystal 1c became bright red under UV-light irradiation for a short irradiation time $(\sim 1 \mathrm{~s})$. Once the UV light was turned off, the compound recovered to its initial state within $1 \mathrm{~min}$. This self-recovery photochromism could be attributed to the solid photocyclization of the TPE moiety under UV-light irradiation. More importantly, its application potential in multidimensional anticounterfeiting for banknotes or confidential paper has been demonstrated, which has been detailed in Section 4.

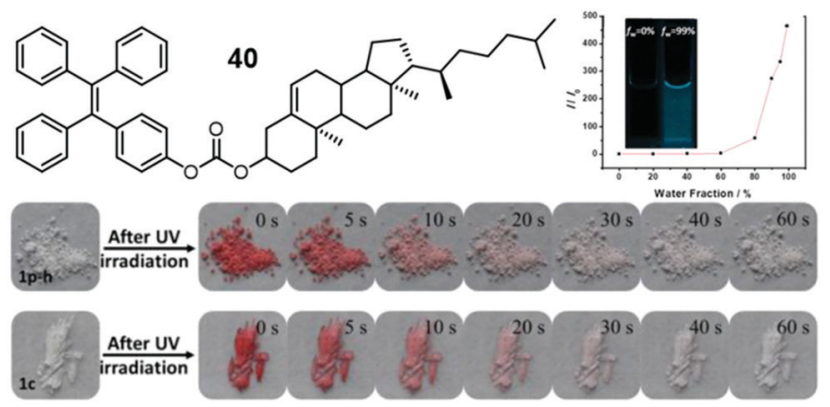

Fig. 15 Chemical structure of 40 . AIE and photochromic behaviors after UV-light irradiation for different values within 0-60 s. Reproduced with permission. ${ }^{49}$ Copyright 2019, Wiley-VCH. 


\section{Morphology of AIE-active photochromic materials}

\subsection{Gels}

Organogels, which self-assemble via noncovalent interactions, have attracted considerable attention in materials science. ${ }^{50}$ As an important branch, smart organogels comprising photochromic units and AIE properties have entered into the spotlight. ${ }^{37,51-54}$ Their morphology and fluorescence can be reversibly controlled via suitable external stimuli and aggregation degree. Therefore, these smart gels have been applied in diverse areas, such as optical memory devices and logic gates.

As shown in Fig. 16A, Park et al. prepared 41a/SS-TFMBE mixed organogel system to evaluate the photochromic performance and compatibility of $\mathbf{4 1 a}$ with these cyanostilbene-based organogelators. ${ }^{51}$ This system exhibited intense fluorescence with the typical AIEE property, and switchable high-contrast dual-mode fluorescence could be achieved (fluorescence switching ratio above 166). Light irradiation induced photochromic isomerization and thermal irradiation controlled the phase transformation between the gel and solution, as shown in Fig. 16A. Thermal irradiation switched on the fluorescence of open-form 41a, while no fluorescence was observed for closedform $41 \mathrm{~b}$ both in the solution and gel states. Such a unique dual-mode-response organogel system could be applied as a binary OR logic gate with "UV light" and "heat" as the input signals and fluorescence off as the output. The resultant truth table is (1) gel/visible light $(0,0),(2)$ gel/UV light $(1,0),(3) \mathrm{sol} /$ visible light $(0,1)$, and (4) sol/UV light $(1,1)$. Moreover, they could achieve high-density optical logic memory storage by

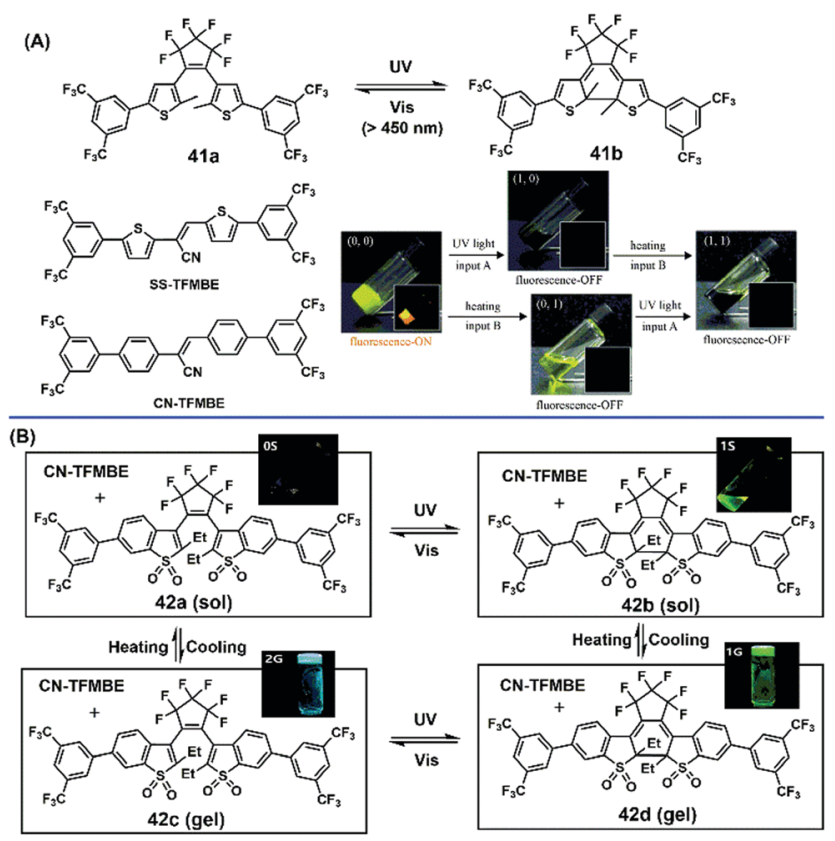

Fig. 16 (A) Photochromic reaction of 41a and illustration of binary logic gate. Reproduced with permission. ${ }^{51}$ Copyright 2009, Wiley-VCH. (B) Schematic illustration of $42 \mathrm{a}$ and photographs of their four states. Reproduced with permission. ${ }^{52}$ Copyright 2018, Wiley-VCH. using this switchable fluorescent organogel system. Further, they could achieve multicolor multistate fluorescence switching system by combining a thermal switch (CN-TFMBE) and a fluorescent switch (DAE 42a), as shown in Fig. 16B. ${ }^{52}$ In the solution state $(\mathbf{O S})$, the combined system showed no fluorescence because of the long distance between the CN-TFMBE molecules and 42a in the 1,1,2,2-tetrachloroethane (TCE) solvent. Under UV-light irradiation, the mixture solution turned from no fluorescence to green fluorescence $(\mathbf{0 S} \rightarrow \mathbf{1 S})$ via a ringclosing reaction. The green fluorescent solution was converted into a green emissive gel upon cooling $(\mathbf{1 S} \rightarrow \mathbf{1 G})$. Then, visible light could induce color switching to blue fluorescence in gel $(\mathbf{1 G} \rightarrow \mathbf{2 G})$. Further heating would bring the $\mathbf{2 G}$ state to return to the OS state with fluorescence quenching. Notably, all these thermoresponsive and photoresponsive fluorescent switches were completely reversible and independent, which could be expressed through integrated logic circuits comprising two different inputs (light and thermal) and three different outputs (on/off green and blue fluorescence and the gel/solution state).

Zhang et al. reported a multiemission colored organogel composed of dendron-substituted TPE (LMWG1) and SP (43a), as shown in Fig. 17. ${ }^{53}$ LMWG1 showed fairly weak fluorescence in toluene but strong fluorescence in the gelation state, which generated a reversible fluorescence switch that could be controlled by heating and cooling. The mixed gels inherited the properties of LMWG1 and 43a, whose emission colors could be switched under alternating UV- and visible-light irradiation and further tuned by varying the irradiation time (Fig. 17B-D) and different molar ratios. The above efforts inspired the further modifications of SP and TPE to obtain better performances and more promising materials for practical applications.

Ran and Guo's group synthesized an organogelator containing 1,3,4-oxadiazole and azobenzene moieties, as shown in Fig. $18 .^{54}$ This gelator exhibited excellent gelation ability in moderate polar solvents and low gelation concentration. Interestingly, under UV-light irradiation for both gel and solution, the morphological transformation from fiber to porous structures was found in dichloromethane via scanning electron microscopy (SEM). Moreover, a significant enhancement in fluorescence was observed followed by the photoisomerization of $\mathbf{4 4}$, which could

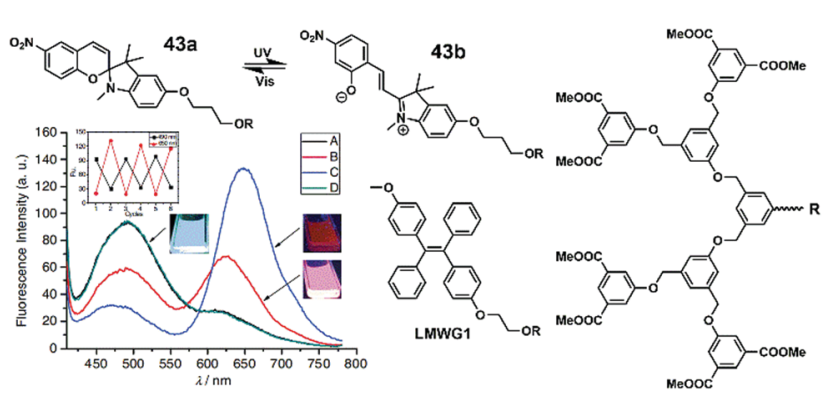

Fig. 17 Structures and fluorescence spectra of the organogel formed with LMWG1 (10 mM) and 43a (10 mM) in toluene (A) after UV-light (365 nm) irradiation for $30 \mathrm{~s}$ (B) and $10 \mathrm{~min}$ (C) and subsequent visible-light (550 nm) irradiation for $10 \mathrm{~min}(\mathrm{D}) ; \lambda_{\text {exc }}=400 \mathrm{~nm}$. Reproduced with permission. ${ }^{53}$ Copyright 2010, Wiley-VCH. 


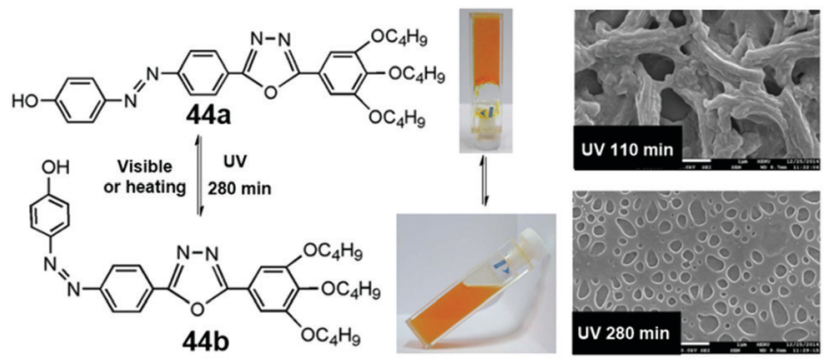

Fig. 18 Structures of $44 a-b$ and their dichloromethane gel $\left(2.0 \mathrm{mg} \mathrm{mL}^{-1}\right)$ before (top) and after (bottom) UV irradiation and the SEM images (right) Reproduced with permission. ${ }^{54}$ Copyright 2015, Royal Society of Chemistry.

be attributed to the arrangement of J-type aggregation and formation of spherical aggregates. These results can facilitate the exploitation of more photoswitchable fluorescent azobenzene derivatives and broadening their application domains.

\subsection{Liquid crystals (LCs)}

LCs are liquid phases possessing some degree of orientational and positional order situated between the crystalline state and liquid state. ${ }^{55}$ These soft materials have been widely utilized in various domains, such as flat-panel liquid crystal display (LCD) devices, multifunctional stimuli-responsive materials, and other advanced functional materials. ${ }^{56}$ Numerous photochromic LCs have been reported based on classic photoswitchable molecules. ${ }^{57}$ However, AIE-active photochromic LCs are still rare.

$\mathrm{Hu}$ and coworkers developed a series of photoresponsive and AIE-active LC gelators $(\mathbf{4 5 - n} ; n=0,1,3,5)$ by connecting a cholesterol moiety and a TPE moiety into the azobenzene moiety with different alkyl spacers, as shown in Fig. $19 .^{58}$ All these 45- $\boldsymbol{n}$ molecules exhibited the smectic A LC phase, but the spacer length could result in different properties. In addition, these molecules possessed significant aggregationinduced and gel-enhanced emissions, and the photocontrolled sol-gel transition was observed. Under UV-light irradiation, the gel state could be converted to the solution state in a DMF solution accompanied by a color change from yellow to orange. As shown in Fig. 19 (right), the textures of the LC phase were clearly observed under polarizing optical microscopy (POM), indicating that the non-rod-like and nonflexible TPE fit well into the rod-like molecular moiety. Such photoresponsive LC gelators with AIE characteristics allowed them to function as chemosensors and optoelectronic materials.

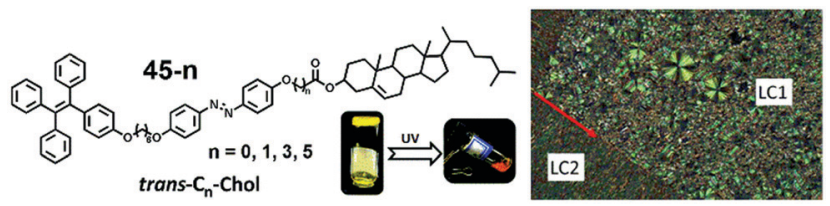

Fig. 19 Structure of 45- $n$ and its photochromic properties and the textures of trans- $\mathrm{C}_{5}$-Chol observed by POM upon cooling from the isotropic phase (right). Reproduced with permission. ${ }^{58}$ Copyright 2018, Royal Society of Chemistry.

\subsection{Polymers}

In order to endow the polymer with photochromic and AIE properties, the most common strategy is to introduce both moieties as the backbone or side chain in the polymer. Park et al. synthesized fluorescent photochromic polymer 46a consisting solely of cyanostilbene-based AIEE fluorophore and DAE units, as shown in Fig. $20 .^{59}$ The weight-average molecular weight $\left(M_{\mathrm{w}}\right)$ of polymer 46a, which was synthesized by the Knoevenagel condensation reaction, was around 13000 $\left(M_{\mathrm{w}} / M_{\mathrm{n}}=2.47\right)$, suggesting the presence of $\sim 7-8$ DAE-cyanostilbene backbone units inside. Moreover, polymer 46a afforded a high glass transition temperature $\left(T_{\mathrm{g}}\right)$ of $188{ }^{\circ} \mathrm{C}$ and exhibited good solubility in common organic solvents. Upon irradiation of the solution or film of polymer 46a under UV light (290 nm), the photoluminescence (PL) emission intensity was significantly diminished, which indicated high-contrast bistable photochromic switching. More importantly, different patterns or information could be successfully written and wiped out on the photochromic polymer film under light exposure on the patterned photomasks, as shown in Fig. 20 (right).

He et al. reported the fabrication of novel AIE-active photochromic polymer 47a (Fig. 21A) via a polycondensation reaction; 47a comprised DAE and TPE moieties. ${ }^{60}$ The original properties of the DAE and TPE moieties were retained in the obtained polymer. The phenyl rings of TPE, which are a part of the backbone units, could still rotate in the THF solution resulting in no emission, and the typical AIE phenomenon would be observed with an increase in the $f_{\mathrm{w}}$ value. The polymer film of open-form 47 a showed an emission at $507 \mathrm{~nm}$, and this fluorescence could be quenched upon $300 \mathrm{~nm}$ UV-light irradiation because of the FRET process from TPE to closed-form DAE. However, subsequent $620 \mathrm{~nm}$ visible-light irradiation would switch on the fluorescence again (Fig. 21A). Such fluorescence switching of 47 a could be repeatedly undertaken, affording excellent fatigue resistance. More recently, another novel photoswitchable polymer 48a containing the DAE unit, triazole linker, and TPE moiety in the polymer backbone was reported by Lin's group; it was used to investigate the AIE behavior and photochromic property. ${ }^{61}$ As shown in Fig. 21B, upon UV-light irradiation, the irreversible photoisomerization from the open to closed forms could occur in pure THF and THF/ $\mathrm{H}_{2} \mathrm{O}$ mixture solution with $f_{\mathrm{w}}$ value of $90 \%$, solid film, and acidic conditions. However, the AIE behavior of the closed form was lost with $f_{\mathrm{w}}$ value of $90 \%$, and a distinctive monomer emission appeared at $385 \mathrm{~nm}$ owing to the efficient energy transfer from the

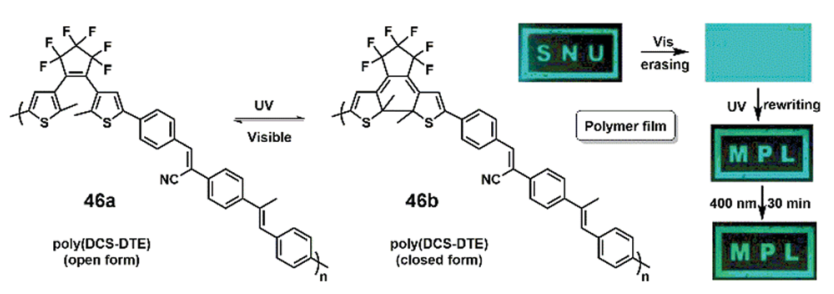

Fig. 20 Photochromic reactions of polymer 46a and erasable fluorescence imaging on a spin-coated polymer 46a film. Reproduced with permission. ${ }^{59}$ Copyright 2005, American Chemical Society. 

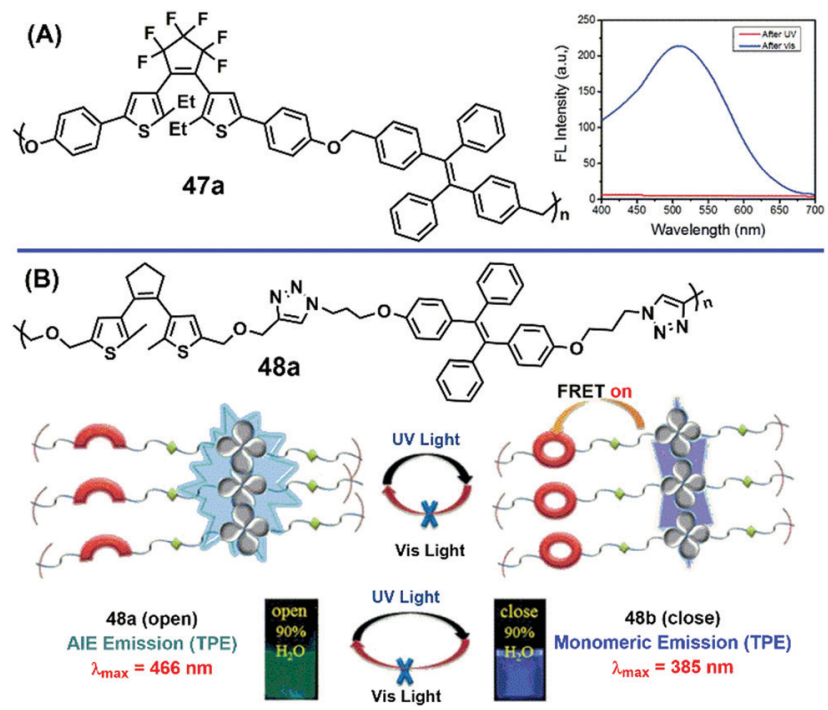

Fig. 21 (A) Structure of polymer $47 a$ and reversible fluorescence switching of the polymer film using UV and visible light. Reproduced with permission. ${ }^{60}$ Copyright 2016, Royal Society of Chemistry. (B) Structure of polymer 48a and pictorial diagram of 48a from its opened to closed form upon UV irradiation. Reproduced with permission. ${ }^{61}$ Copyright 2017, Royal Society of Chemistry.

TPE to the ring-closed DAE moiety. Interestingly, such photocyclization of 48a induced a significant decrease in AIE emissions and increase in monomer emissions, which were observed for the first time and offered a special viewpoint on the photoswitchable AIE molecules.

Later, Luo's group modified tetraarylethene with a pyridine group to fabricate a coordination polymer that was connected via the coordination of $\mathrm{ZnCl}_{2}$ with pyridine moieties, as shown in Fig. 22. ${ }^{62}$ Both monomer 49a and polymer 50a exhibited typical AIE characteristics in a $\mathrm{H}_{2} \mathrm{O} / \mathrm{THF}$ mixed solution. Upon alternate UV- and visible-light irradiation, reversible photoisomerization reactions of 49a and 50a could occur both in the solution and solid states.

Furthermore, Zhou and Zhu's group successfully prepared amphiphilic diblock copolymer $\mathbf{5 1}$ by using the reversible addition-fragmentation chain-transfer (RAFT) polymerization technique (Fig. 23A).$^{63}$ Because of the isomerization of azobenzene, the absorption peak related to the trans form at $420 \mathrm{~nm}$ decreased, while the peak related to the cis form at $550 \mathrm{~nm}$ gradually increased upon UV-light irradiation. Typical AIE phenomenon was observed in a water/THF mixed solution of copolymer 51. Later, TPE-based polymers with azobenzene pendants were prepared by He's group via a post-polymerization azo-coupling scheme, as shown in Fig. 23B. ${ }^{64}$ The functional degree of azobenzene chromophores exhibited good relationship with the feeding ratio, which could significantly affect their emission behaviors. The fluorescence of polymer $\mathbf{5 2}$ could be quenched due to the FRET process from the TPE moiety to the azobenzene part. By the reduction of azobenzene to hydrazobenzene in polymer 52, the fluorescence of TPE could recover again, which indicated a redox-reaction-triggered fluorescence process.

Lin et al. reported novel amphiphilic AIE copolymer 53a containing the TPE-SP pendant as a fluorophoric side chain. ${ }^{65}$

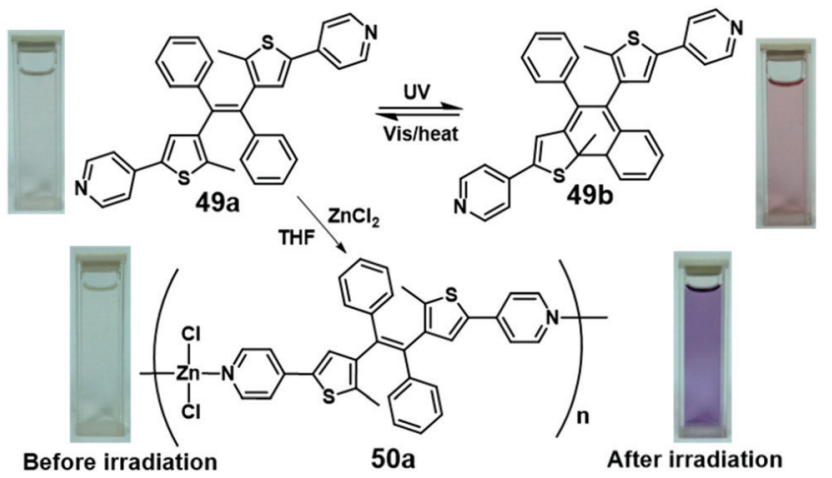

Fig. 22 Photochromic reactions and correlative color changes of monomer 49a and polymer 50a. Reproduced with permission. ${ }^{62}$ Copyright 2018 , Chemical Society of Japan.

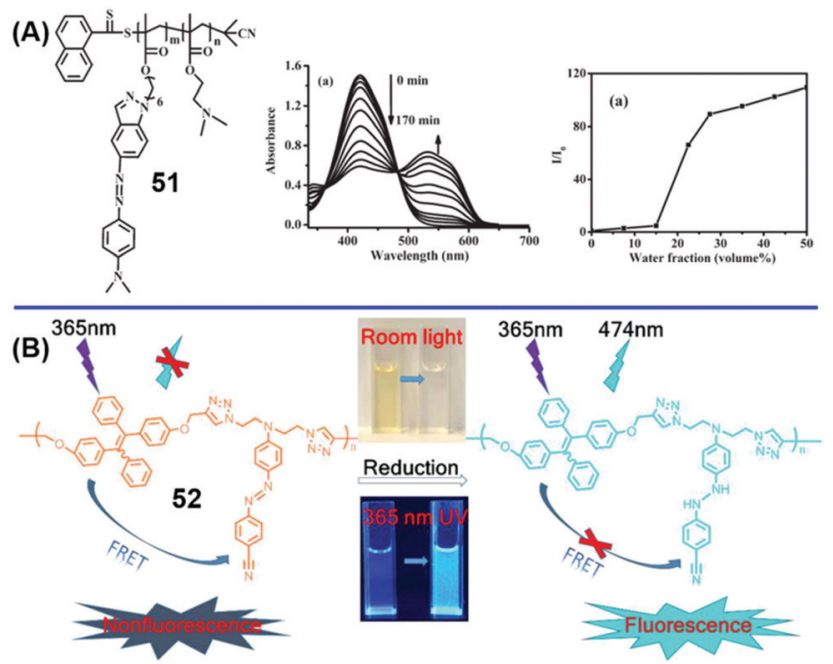

Fig. 23 (A) Structure, UV-vis spectra, and AIE properties of copolymer $\mathbf{5 1 .}$ Reproduced with permission. ${ }^{63}$ Copyright 2011, Wiley-VCH. (B) Redoxreaction-triggered process of polymer $\mathbf{5 2}$. Reproduced with permission. ${ }^{64}$ Copyright 2018, Elsevier.

As shown in Fig. 24, closed-form 53a exhibited strong green fluorescence in an aqueous solution. Upon UV-light irradiation, it switched to the open-form $\mathbf{5 3 b}$ of strong red fluorescence

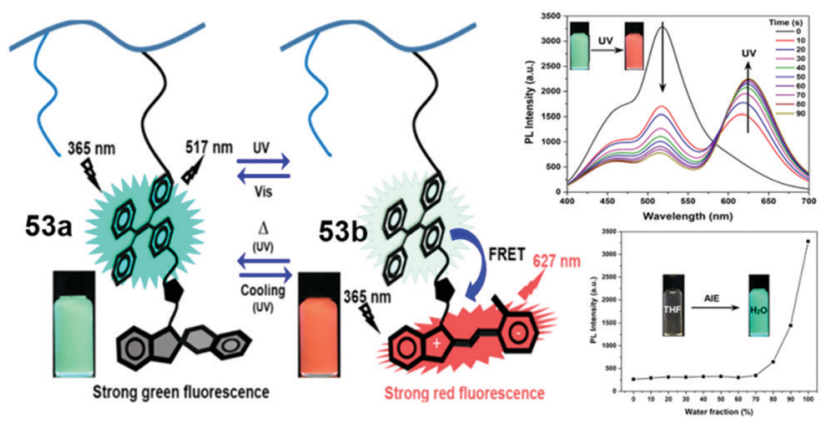

Fig. 24 Schematic illustration of the energy transfer from the TPE to MC unit via the FRET process after UV exposure and the AIE property of 53a in $\mathrm{THF} / \mathrm{H}_{2} \mathrm{O}$ solutions. Reproduced with permission. ${ }^{65}$ Copyright 2020 , American Chemical Society. 
merocyanine accompanied by the FRET process. The PL emission intensity of copolymer 53a gradually increased in $\mathrm{THF} / \mathrm{H}_{2} \mathrm{O}$ solutions with increased $f_{\mathrm{w}}$ and the maximum emission was reached at $100 \% f_{\mathrm{w}}$; the inset shows the non-emission and green emission in pure THF and water, respectively. In addition, they performed deeper investigations with respect to various conditions, such as $\mathrm{pH}$, thermal, and anionic detection. This copolymer showed good biocompatibility and high selectivity for $\mathrm{CN}^{-}$detection.

\subsection{Nanoparticles (NPs)}

Park et al. introduced 1-cyano-trans-1,2-bis-(4'-methylbiphenyl)ethylene (CN-MBE) onto photochromic DAE (54a) as the AIEE group. ${ }^{66}$ As shown in Fig. 25A, rather weak fluorescence was displayed in the THF solution of $\mathbf{5 4 a}$, but strong fluorescence was observed in the state of fluorescent photochromic organic nanoparticles (FPONs). However, only about $35 \%$ of the open form (54a) was photoisomerized into the closed form (54b) in the PSS of its $\mathrm{CDCl}_{3}$ solution $\left(2 \times 10^{-4} \mathrm{M}\right)$ upon light irradiation at $365 \mathrm{~nm}$. In case of the FPONs state of 54a, the conversion ratio in the PSS was even smaller because the ring-closing reaction could occur only at the surfaces of the FPONs. In the sizetuned neat NPs of 54a and 54a-loaded PMMA film, on-off-type fluorescent switches were successfully demonstrated under high contrast $(>10)$. Moreover, Wang et al. tried to connect a similar AIE fragment to both the sides of a DAE unit via a covalent bond for further investigations, as shown in Fig. 25B.$^{67}$ They found that the trans-to-cis isomerization of the cyanoethylene units occurred prior to the DAE isomerization. In addition, the AIE behavior was investigated in the $\mathrm{THF} / \mathrm{H}_{2} \mathrm{O}$ system, and nanowires were generated by adding $60 \mathrm{vol} \%$ fraction of water, which could be confirmed by field-emission scanning electron microscopy (FE-SEM). The nanowires possessed intense blue luminescence and could gradually melt away by increasing the time of irradiation ( $365 \mathrm{~nm}$ light).

As typical AIEgens, the TPE molecule has drawn considerable attention for its facile preparation and modification
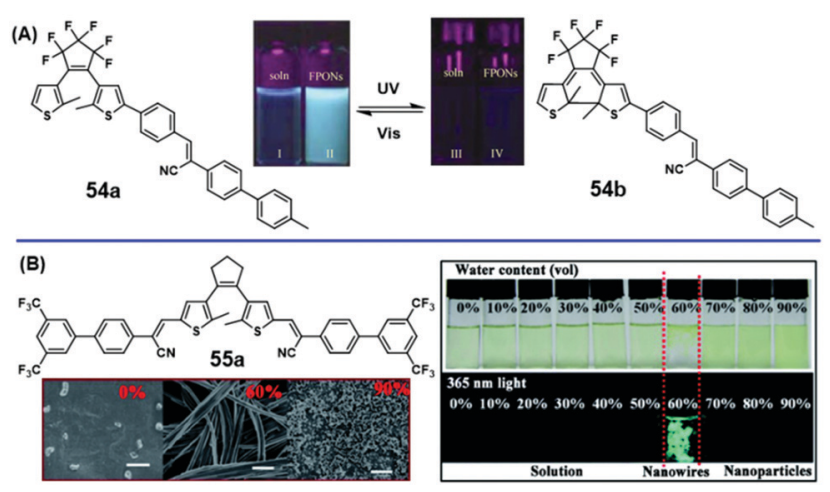

Fig. 25 (A) Chemical structure of 54a and the fluorescence images of its THF solution (I, soln, $2 \times 10^{-4} \mathrm{M}$ ) and the colloidal suspension (II) of the FPONs $\left(2 \times 10^{-4} \mathrm{M}\right)$. Reproduced with permission. ${ }^{66}$ Copyright 2004 , Wiley-VCH. (B) Structure of $\mathbf{5 5 a}$; photographs of $\mathbf{5 5 a}$ in solution with different volume proportions of THF and $\mathrm{H}_{2} \mathrm{O}$; and $\mathrm{FE}-\mathrm{SEM}$ images of aqueous $0 \%, 60 \%$, and $90 \%$ samples (volume percentage of water). Reproduced with permission. ${ }^{67}$ Copyright 2018, Science China Press.
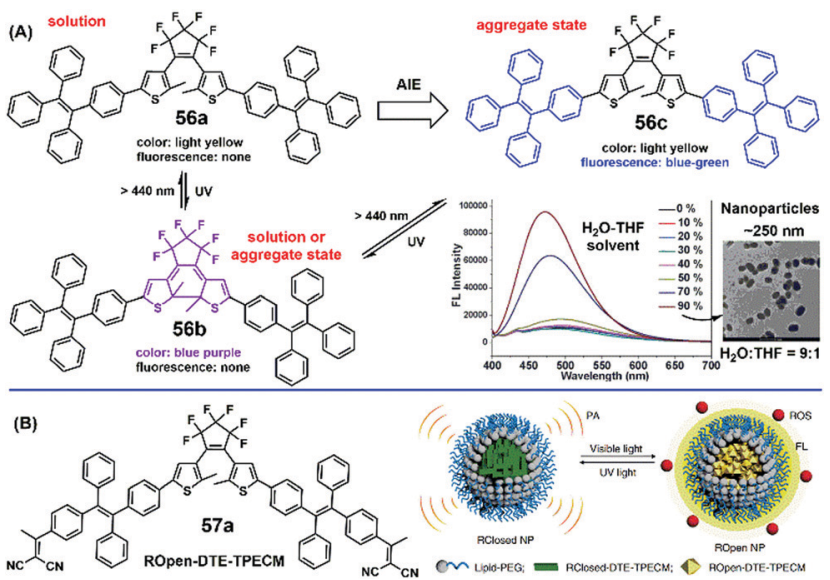

Fig. 26 (A) Photochromism and fluorescence on/off in solution and the "aggregate" state of $\mathbf{5 6 a - c}$; emission spectra of 56a in different THF/ $\mathrm{H}_{2} \mathrm{O}$ solvent mixtures (concentration: $1 \times 10^{-4} \mathrm{M}$; excitation: $385 \mathrm{~nm}$ ). The inset shows the TEM image after evaporation of $90 \%$ water as a NP. Reproduced with permission. ${ }^{68}$ Copyright 2013, Royal Society of Chemistry. (B) Structure of $57 \mathrm{a}$ and schematics of RClosed and ROpen NPs. Reproduced with permission. ${ }^{69}$ Copyright 2018, Nature Publishing Group.

abilities, excellent thermal properties, and high fluorescence quantum yields. ${ }^{21 b}$ As shown in Fig. 26A, Zhu et al. were the first to report TPE-based photochromic material 56a, which quenched its fluorescence in a solution but exhibited enhanced blue-green fluorescence in the solid state and NPs. ${ }^{68}$ After $365 \mathrm{~nm}$ UV-light irradiation, the blue-green fluorescence at $520-540 \mathrm{~nm}$ gets quenched with $90 \%$ quenching efficiency in PSS (56b). In addition, the inset in Fig. 26A shows that the fluorescence intensity gradually increases with an increase in the $f_{\mathrm{w}}$ value, suggesting the AIE characteristic of 56c. NPs obtained at higher $f_{\mathrm{w}}$ values showed a subsequent enhanced emission owing to the restriction of the internal bond motions of the TPE moiety. In particular, a spin-coated PMMA film of 56a was successfully utilized for super-resolution fluorescence imaging, which was clearer and more distinguishable than the conventional one. Later, Qi et al. introduced a similar molecule (57a) consisting of a DAE switch and two surrounding 2-(1-(4(1,2,2-triphenylvinyl)phenyl)ethylidene)malononitrile (TPECM) units, as shown in Fig. 26B. ${ }^{69}$ 57a exhibited AIE characteristics in a $\mathrm{THF} / \mathrm{H}_{2} \mathrm{O}$ system. However, the closed form showed no detectable fluorescence emission in the THF solution as well as the aggregate state due to the intramolecular energy transfer process from the fluorescent ТРЕСM to the nonemissive closed-form DAE moiety. Moreover, the ring-closed and ring-opened NPs were prepared by a nanoprecipitation method using amphiphilic maleimide-bearing $\mathbf{P E G}_{2000}$ lipid as the doping matrix, which was surrounded by a hydrophilic outer layer of PEG to stabilize the NPs, as shown in Fig. 26B (right). The ring-closed and ring-opened NPs exhibited highly reversible and bistable photochromism properties under alternating UV-/visible-light irradiation. More interestingly, the NPs could be further functionalized and served as a photosensitizer, versatile photoacoustic (PA) contrast agent, and fluorescent probe. 
Qian's group synthesized a special class of binary composite nanoparticles (CNPs) by the reprecipitation method in a THF/ $\mathrm{H}_{2} \mathrm{O}$ mixed solution based on photochromic BTF6 and fluorescent DHBIA molecules, as shown in Fig. 27A. ${ }^{70}$ A strong fluorescence signal was obtained with the CNPs due to the employment of DHBIA molecules, which showed remarkable AIEE in the solid state. In addition, the fluorescence of the CNPs could be switched on/off under alternating UV-/visiblelight irradiation because of the photochromic 58a component. Moderate-contrast fluorescence on/off switching could be obtained because of the intermolecular energy transfer from the DHBIA molecules to the ring-closed form of 58a. Furthermore, these CNPs were also successfully applied in PVA films with good fatigue resistance. Besides, the coumarin fluorescent unit could also be connected with photochromic dithienylethenes to afford 59a, which represented a unique example of the AIE characteristic. ${ }^{71}$ As shown in Fig. 27B, very weak fluorescence was produced in the THF solution, but it became stronger by adding water, which could be attributed to the formation of NPs via the hydrophobic interactions of dithienylethene moieties. Under $254 \mathrm{~nm}$ UV-light irradiation, the fluorescence was continuously enhanced by the enhanced aggregation of the more rigid closed form of 59b.

As shown above, a lot of efforts have been made toward fabricating AIE-active photochromic NPs based on small molecules; a few reports have discussed the applications of AIE fluorophores in photoswitchable fluorescent polymer nanoparticles (PFPNs). Chen et al. prepared PFPNs by using an AIE fluorophore (p-DTPACO) and an amphiphilic copolymer (60a) containing photochromic SP as the side chain via the coprecipitation technique. ${ }^{72}$ This AIE-active p-DTPACO could not only
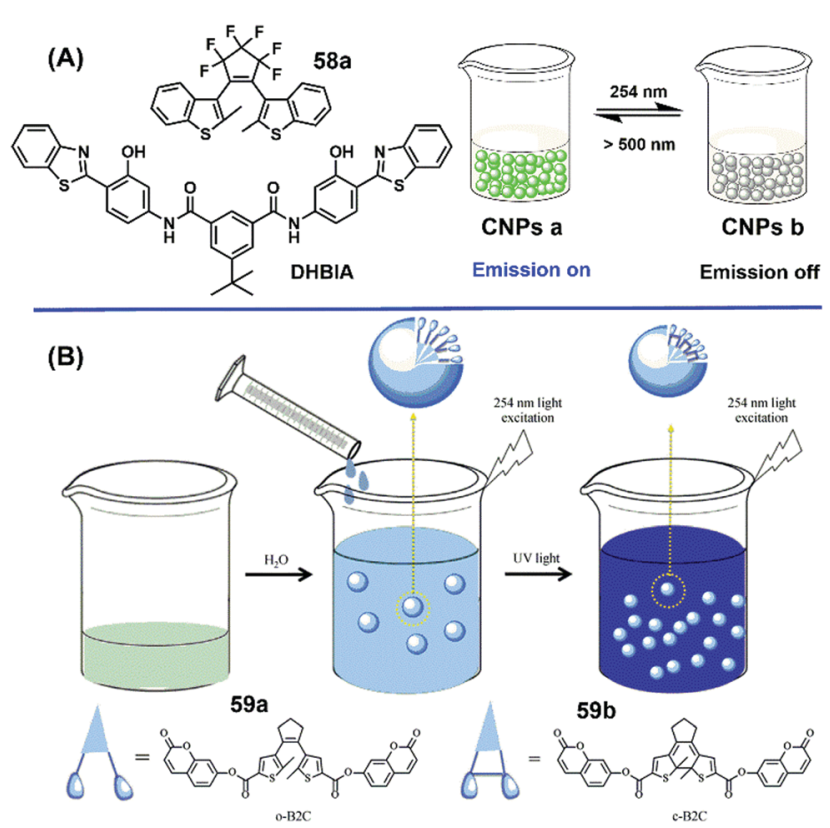

Fig. 27 (A) Mechanism of fluorescence switching in CNPs. Reproduced with permission. (B) Schematic representation of the morphology and fluorescence transition by the addition of water and UV-light irradiation. Reproduced with permission. ${ }^{71}$ Copyright 2015, Elsevier. effectively prevent the notorious ACQ problem, but also could be considered as an effective fluorescent donor, as shown in Fig. 28A. In addition, the ring-opened isomer of SP could quench the fluorescence of the donor, which would result in a high-contrast dual-color fluorescence signal. Amphiphilic diblock copolymer 60a containing the SP moieties were vital because they were not only reversible switches but they also acted as carriers to stabilize the hydrophobic AIE-active p-DTPACO. More importantly, the resultant PFPNs were suitable for rewritable data storage, rewritable printing, or imaging applications. More recently, Feng et al. synthesized a series of NPs by integrating SP-containing copolymers $\mathbf{6 1 b}$ with plasmid DNA via electrostatic interactions. ${ }^{73}$ In copolymer $\mathbf{6 1 b}$ (Fig. 28B), the imidazole units acted as the DNA binding and condensing sites to form similar morphologies of NPs, and the other units also had an important role in this work. The formed NPs were stable in buffer solutions containing salt or serum, indicating good biocompatibility. Besides, the efficient reversible photoisomerization of $\mathbf{6 1 b}$ NPs was observed with good fatigue resistance when irradiated by alternate UV-/visible-light irradiation. Interestingly, upon the activation of MC units by visible light, both copolymer $\mathbf{6 1 b}$ and NP of $\mathbf{6 1 b}$ could be used as a photosensitizer for ${ }^{1} \mathrm{O}_{2}$ generation in aqueous solutions

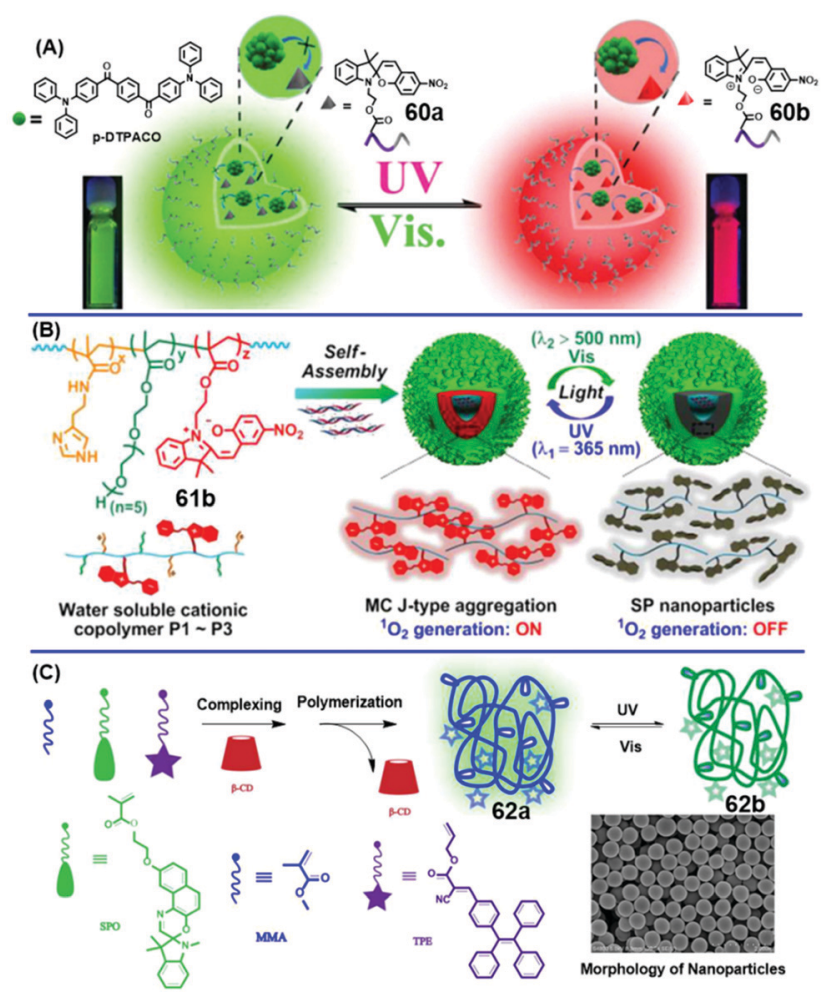

Fig. 28 (A) Schematic illustration of the photoswitching behavior of PFPNs under UV- and visible-light irradiation. Reproduced with permission. ${ }^{72}$ Copyright 2017, Royal Society of Chemistry. (B) Self-assembly of polymers $\mathbf{6 1 b}$ with DNA, and reversible photochromic reaction. Reproduced with permission. ${ }^{73}$ Copyright 2019, Royal Society of Chemistry. (C) Preparation and morphology of NP 62a and its photochromic reactions. Reproduced with permission. ${ }^{32}$ Copyright 2017, Elsevier. 
and cells. This concept provided a novel strategy toward the design of reversible and controllable AIE-active photochromic systems.

Wang's group developed photochromic fluorescent NP 62a via the semi-continuous polymerization of methyl methacrylate (MMA), SO-decorated MMA, and TPE-decorated MMA, as shown in Fig. 28C. ${ }^{32}$ The polymerization occurred in water under the presence of methylated $\beta$-cyclodextrin $(\beta-C D)$, which played important roles in increasing the polymerization efficiency and forming uniform NP 62a. In addition, the NP exhibited the AIE characteristics and its fluorescence could be reversibly switched on/off under alternate UV- and visible-light irradiation, which can find applications in biological fluorescent labeling as well as other fluorescent fields.

Li and Zhu's group fabricated new supramolecular functional NP 63 via the self-assembly of a supramolecular amphiphile formed by the guest-host interactions between the DMAECD host and DMA-Azo-AD guest, as shown in Fig. $29 .^{74}$ This new supramolecular NP inherited the properties of dual $\mathrm{pH} /$ photoresponsiveness and AIE behavior, ${ }^{75}$ and it displayed the ability to achieve a unique combination of dynamic morphological transition and reversible AIE-active fluorescent switching triggered by $\mathrm{pH}$ or light stimuli. Moreover, an effective DNA condensation ability with extremely low cytotoxicity was demonstrated by this system, which suggested its potential applications in efficient in vitro gene delivery and bioimaging.

\subsection{Supramolecular systems}

Wang's group reported the fluorescence photoswitching system $\mathbf{G} \subset \mathbf{H}$ comprising a TPE-based pillar[5] arene host $(\mathbf{H})$ and a photoresponsive BTE guest G (or 64a), as shown in Fig. $30 .^{76}$ The fluorescence of $\mathbf{G} \subset \mathbf{H}$ could be switched on/off via the photoisomerization of the BTE unit both in the solution and solid states under alternate UV-/visible-light irradiation. The fluorescence intensity of $\mathbf{G} \subset \mathbf{H}$ was considerably enhanced with $f_{\mathrm{w}}$ values of $50 \%$ and $60 \%$ in $\mathrm{THF} / \mathrm{H}_{2} \mathrm{O}$ mixed solutions when assembled with $\mathbf{G}$. Such fluorescence enhancement could be ascribed to the restrictions in the intramolecular motions of TPE by the host-guest interactions between one $\mathbf{G}$ molecule and two $\mathbf{H}$ molecules. Besides, $\mathbf{G} \subset \mathbf{H}$ could self-assemble into round-cake-like nanostructures when the $f_{\mathrm{w}}$ value was $60 \%$, which would break into shuttle-like nanostructures under UV-light irradiation. In addition, the PMMA film loaded with

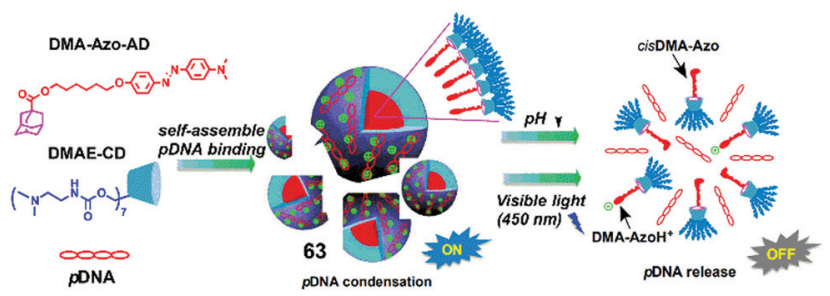

Fig. 29 Schematic illustration of a dual-responsive supramolecular nanoparticle formed by the self-assembly of a supramolecular amphiphile, and its $\mathrm{pH}$ - or visible-light-triggered pDNA release as well as fluorescence quenching behavior. Reproduced with permission. ${ }^{74}$ Copyright 2016 , Royal Society of Chemistry.

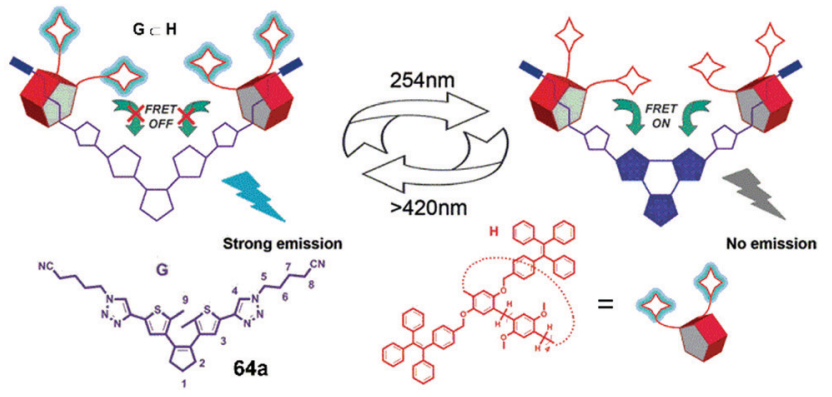

Fig. 30 Chemical structures of the AlE-active pillar[5]arene host $\mathbf{H}$ and dithienylethylene guest $\mathbf{G}(\mathbf{6 4 a})$ and the schematic representation of the photoresponsive fluorescence switching process of the complexes of $\mathbf{H}$ and G. Reproduced with permission. ${ }^{76}$ Copyright 2018, Royal Society of Chemistry.

the $\mathbf{G} \subset \mathbf{H}$ complex was successfully applied in rewritable printing and recording, and there was no obvious loss after repeating the recording/erasing cycles for several times.

More recently, Mukherjee et al. reported the first example of self-assembled macrocycles 65 that exhibited multifunctions of photochromic, acidochromic, and AIE abilities. ${ }^{77}$ As shown in Fig. 31, macrocycle 65 was rationally designed via the coordination of AIE-active Pt(II) acceptor a2 with SP-decorated triarylamine-based dipyridyl donor 65a. Even though the macrocycle was formed, the AIE behavior of building block a2 was still retained and observed in a DCM/hexane mixed solution. In addition, photochromic reversibility was investigated in the presence of alternate UV-/visible-light irradiation, affording a yellow and green color conversion. Moreover, the acidochromism could be observed by using external deprotonation on the open form. Therefore, this self-assembled macrocycle of 65 was demonstrated as a multi-stimuli-responsive system (photochromic, acidochromic, and AIE), which provided a novel strategy for designing multifunctional materials.

Liu et al. constructed a novel crosslinked supramolecular polymer network based on the ternary host-guest molecular recognition motif between cucurbit[8]uril (CB[8]), TPE-based

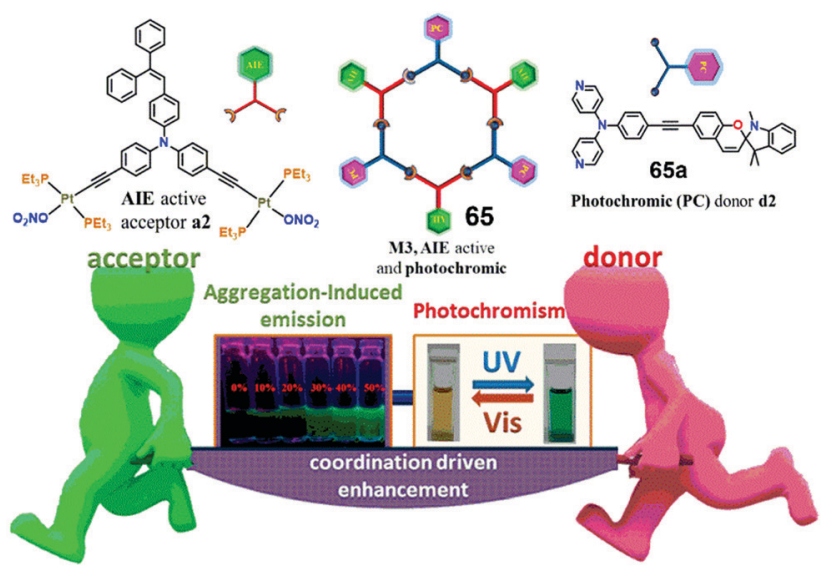

Fig. 31 Structures of building blocks a2, d2 (65a), and macrocycles 65 and the AIE-active photochromic properties of 65 . Reproduced with permission. ${ }^{77}$ Copyright 2019, American Chemical Society. 


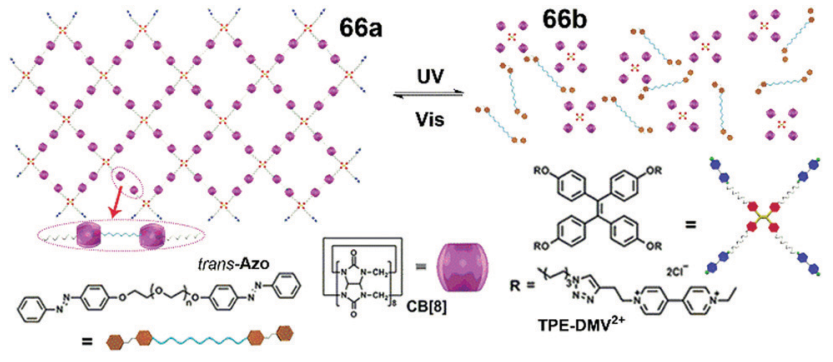

Fig. 32 Chemical structures and schematic representations of the photocontrolled formation of crosslinked supramolecular polymer 66a. Reproduced with permission. ${ }^{78}$ Copyright 2016, Royal Society of Chemistry.

1,1-dimethyl-4,4-bipyridinium dication (TPE-DMV ${ }^{2+}$ ), and azobenzene derivative (trans-Azo) in water, as shown in Fig. 32. ${ }^{78}$ Supramolecular polymerization took place in water, and the obtained supramolecular polymer of 66a exhibited stronger fluorescence than its monomer $\mathbf{6 6 \mathbf { b }}$ because of the AIE effect. More interestingly, the azobenzene moiety could serve as a photoswitch for the reversible polymer-oligomer transitions upon alternating UV- and visible-light irradiation. This was the first reported work providing a new concept for the designing of cucurbituril-based fluorescent materials.

\section{Application of AIE-active photochromic materials}

\subsection{Data recording and storage}

Due to the ability of photoinduced reversible changes between two distinct isomers, it is well known that photochromic materials are promising candidates for data recording and storage applications in the photon mode. ${ }^{79}$ Since the data recording, storage, and readout mechanisms are based on the photoisomerization of two isomeric states, the following properties are required: (1) good stability of both isomers, (2) excellent fatigue resistance for cyclic writing and erasing processes, (3) fast response to the light stimuli, and (4) high resolution. Moreover, the solid or aggregate state is often required for practical applications. ${ }^{79,80}$ Therefore, the introduction of the AIE characteristic into photochromic molecules can be helpful and prove to be an efficient strategy for developing practical data recording and storage materials: a lot of efforts have been dedicated toward this field..$^{22,23,28,59,81}$ Via light irradiation on a specially molded mask, the corresponding patterns could be recorded on a polymer film loaded with photochromic molecules. Next, the erasing process could be operated by the further irradiation of all the films, and another pattern could rewrite the films. As an example (shown in Fig. 33), Liu's group ${ }^{81}$ designed dithienylethene-bridged bispyridinium salt 67a that exhibited outstanding photochromic property with AIE in both organic solution and solid state, as expected. Importantly, the PVDF and PMMA films of 67a showed good reproducibility and easier operability. When the dragon-shaped mask covered the films, the exposed part could be converted to closed-form $\mathbf{6 7 b}$ under fluorescence quenching

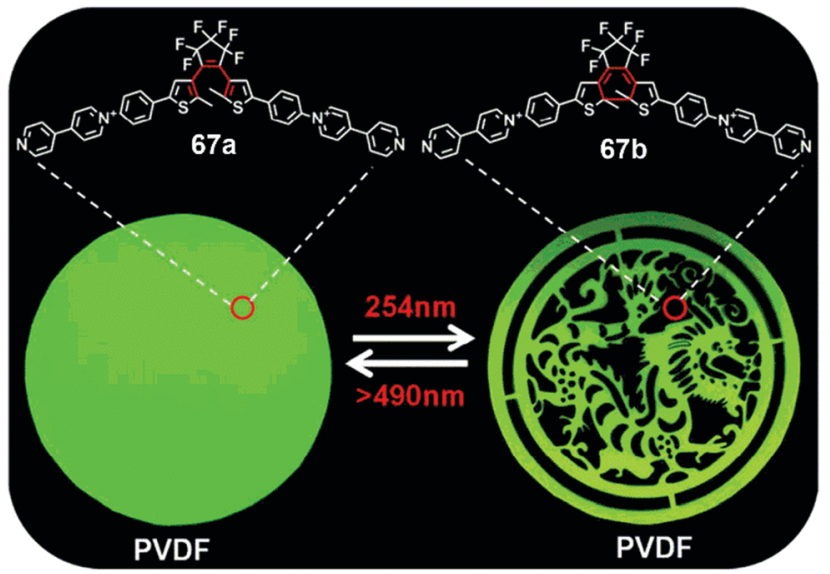

Fig. 33 Fluorescent photographs of 67a-embedded PVDF film under alternating $254 \mathrm{~nm}$ UV- and visible-light irradiations. Reproduced with permission. ${ }^{81}$ Copyright 2018, American Chemical Society.

via UV-light irradiation. The mask-sheltered part maintained its original fluorescence of the open form to create a dragonshaped pattern. Visible light at $490 \mathrm{~nm}$ could assist the printed film to get completely erased, which could be attributed to the transformation of the open form.

\subsection{Logic gates}

It is well known that photochromic molecules can be reversibly switched to two different fluorescent states, which can eventually be used as binary " 1 " and " 0 " in logic gates. ${ }^{18,82}$ AIE materials exhibit strong fluorescence in the aggregate state, but they have weak or no fluorescence in a solution. Therefore, a combination of both these materials in a single system can be an efficient approach to achieve reversible multistate fluorescence switching under light and aggregation stimuli. This kind of AIE-active photochromic materials are naturally useful for logic gate applications. ${ }^{51,52}$

As shown in Fig. $34,{ }^{52}$ there is no fluorescence in the solution state (0S). Upon UV-light irradiation, the nonfluorescent solution emitted green fluorescence (1S) via a photoisomerization reaction $(\mathbf{0 S} \rightarrow \mathbf{1 S})$. The green fluorescent solution was converted into a green emissive gel upon cooling $(\mathbf{1 S} \rightarrow \mathbf{1 G})$. Then, the visible light could drive color switching to blue fluorescence in

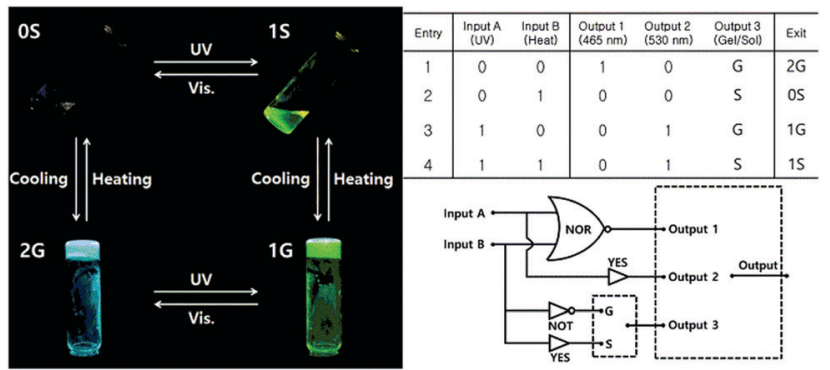

Fig. 34 Photographs of the four states (2G, 0S, 1G, and 1S) (right), truth table (right top), and schematic illustration of the organogel-based integrated logic circuit (right bottom). Reproduced with permission. ${ }^{52}$ Copyright 2018, Wiley-VCH. 
the gel $(\mathbf{1 G} \rightarrow \mathbf{2 G})$. Further heating would bring the $\mathbf{2 G}$ state to return to the $\mathbf{0 G}$ state with fluorescence quenching. Taking advantage of this reversible multistate fluorescence switching, two different inputs (input $\mathbf{A}$ or $\mathbf{B}$ under light or thermal stimuli, respectively) and three distinguishable outputs (outputs 1 and 2 as the on/off green and blue fluorescence and output 3 as the gel/solution state) could find applications as a combinational logic circuit.

\subsection{Super-resolution fluorescent imaging}

Recently, super-resolution fluorescent imaging has proven to be an efficient tool to visualize the structures of biomolecules at the nanometer scale. Photoswitchable fluorophores that can break the diffraction limit play an important role in this field. ${ }^{83}$ In addition, AIE fluorogens have been widely utilized in the biological field due to the strong fluorescence intensity in the aggregate state, and AIE-involving imaging systems exhibited better optical properties than conventional organic dyes. ${ }^{84}$ Hence, the introduction of the AIE characteristic into photoswitchable fluorophores can be a desirable and powerful strategy to develop promising super-resolution fluorescent imaging agents.

However, the combination of photoswitchable molecules with AIE in this field has been rarely reported. ${ }^{30,85}$ Here, we show an example from Zhu's group (Fig. 35). ${ }^{85}$ They prepared sterically hindered DAE systems by substituting with different sterically hindered side groups that exhibited different flexibleness of molecular conformation. Under this rational design, they investigated the dual AIE behavior and demonstrated how AIE activity could be tuned in a sterically hindered photochromic DAE system. More importantly, an excellent lightdriven off-to-on fluorescent switching system with outstanding
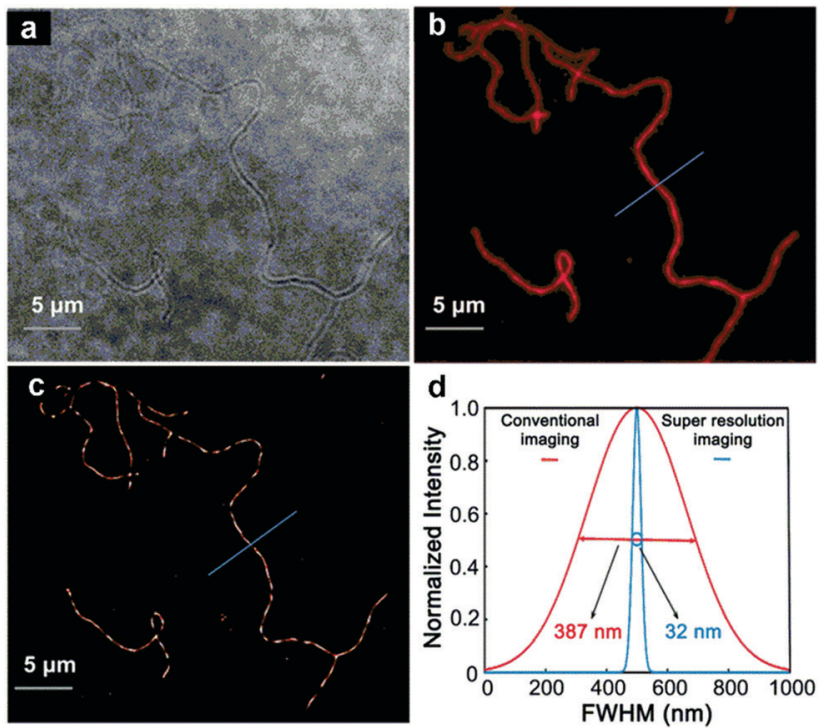

Fig. 35 Super-resolution imaging of block copolymer micelles. (a) Brightfield image. (b) Conventional fluorescence image. (c) Super-resolution image corresponding to the same field of the fluorescence image. (d) Crosssectional profiles of conventional fluorescence and super-resolution imaging. Reproduced with permission. ${ }^{85}$ Copyright 2019, Wiley-VCH.
AIE performance was fabricated and successfully applied in super-resolution imaging. As shown in Fig. 35c, compared with conventional fluorescent imaging (Fig. 35b), the reconstructed fluorescent images exhibited remarkably enhanced resolution. Unsurprisingly, the full-width at half-maximum in superresolution imaging was about $32 \mathrm{~nm}$, which was a 12-fold enhancement than the conventional one (Fig. 35d). Such an AIE-active photochromic system provided a prospective model design for a super-resolution imaging agent.

\subsection{Chemosensors}

Photochromic molecules have been widely utilized in chemosensors owing to their reversible dual-color or fluorescence on-off switching upon appropriate light irradiation. ${ }^{86}$ The introduction of the AIE feature into photoswitchable fluorescent sensors can not only overcome the notorious ACQ problem, but also improve the fluorescence intensity to remove interference from the background. ${ }^{87}$

So far, only a few AIE-active photoswitchable fluorescent chemosensors have been designed. ${ }^{29,65,88}$ As an example shown in Fig. 5C, 16a exhibited multi-stimuli-responsive property with multiple colors in the solid and solution states. The $\mathrm{pH}$ value and sulfite-anion detection ability could be achieved with good optical signals. ${ }^{29}$ Besides, Zhang et al. synthesized a new photoswitchable AIE nanoprobe (68) for lysosomal $\mathrm{H}_{2} \mathrm{~S}$ detection via a combination of $\mathrm{H}_{2} \mathrm{~S}$-recognizing AIE group with photochromic SP moiety, as shown in Fig. 36A. ${ }^{88}$ The mechanism for sensing $\mathrm{H}_{2} \mathrm{~S}$ was that the 2,4-dinitrobenzenesulfonate ester could be cleaved from $\mathbf{6 8}$ to generate AIE probe $\mathbf{6 8 a}$ with a stronger emission at $592 \mathrm{~nm}$. Importantly, a negligible interfering effect on $\mathrm{H}_{2} \mathrm{~S}$ detection could be achieved under existing environment with various anions, and the detection limit was determined to be $5 \mathrm{nM}$. Moreover, the $\mathrm{H}_{2} \mathrm{~S}$-activated AIE

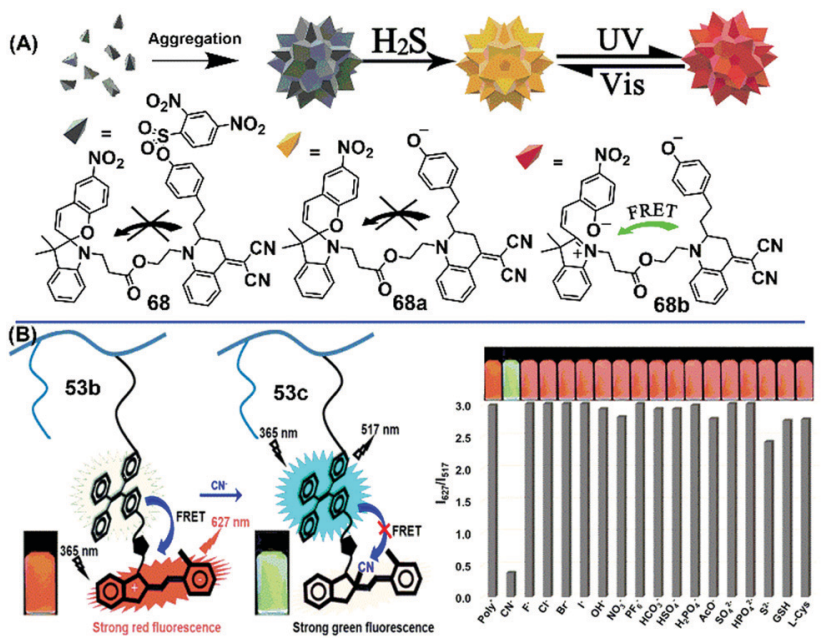

Fig. 36 (A) Overall strategy for 68 for sensing lysosomal endogenous $\mathrm{H}_{2} \mathrm{~S}$ and reversible dual-color imaging. Reproduced with permission. ${ }^{88}$ Copyright 2018, Elsevier. (B) Proposed schematic illustration for the $\mathrm{CN}^{-}$ detection mechanism and fluorescence intensity ratios for the response of $\mathbf{5 3 b}$ upon the addition of various analytes (200 $\mu \mathrm{M})$. Reproduced with permission. ${ }^{65}$ Copyright 2019, American Chemical Society. 
nanoprobe (68a) could be reversibly switched under alternating UV-/visible-light irradiation via the FRET process from the AIE fluorophore to the open form of SP. Recently, Lin et al. reported novel amphiphilic AIE copolymer 53a, where open-form 53b could be used as an amphiphilic chemosensor for $\mathrm{CN}^{-}$detection, as shown in Fig. 36B. ${ }^{65}$ The nucleophilic addition of $\mathrm{CN}^{-}$ with the carbon atom in the open form could block the FRET process resulting in the recovery of green fluorescence from the TPE. Moreover, this chemosensor showed higher selectivity and sensitivity toward $\mathrm{CN}^{-}$in water, which could be utilized in environmental and biological systems.

\subsection{Anticounterfeiting}

Information security has gained paramount importance in our daily lives. Among all kinds of anticounterfeiting technologies, fluorescent ones play an essential role owing to their higher concealment nature. ${ }^{89}$ Meanwhile, photoswitchable fluorescent molecules in the solid or aggregate states that possess the reversible luminescence modulation property are highly attractive in many advanced applications. The utilization of photoswitchable fluorescent molecules has been considered to be a promising but challenging concept in the anticounterfeiting domain, and publications in this regard are still rare. ${ }^{49}$ Tang's group designed TPE derivative 40 that integrated AIE, self-recovery photochromism, and multistate mechanochromism, as shown in Fig. 15. They combined and exhibited multi-fluorescent states of 40 on an example banknote to evidence the potential applications in anticounterfeiting (Fig. 37). The rose pattern was almost invisible under natural light. Upon UV-light irradiation, a blue background, dim petals, cyan leaves, and rose contour became visible. A further increase in the UV-light intensity could facilitate the reddening of the petals. After turning off the UV light, the bright red rose gradually disappeared with a recovery to the initial state. Similarly, some additional personalized patterns could be designed and printed in light of specific requirements.

\section{Summaries and perspective}

Recently, novel classes of AIE-active photochromic materials have been reported, and considerable efforts have been continuously dedicated toward developing more materials for

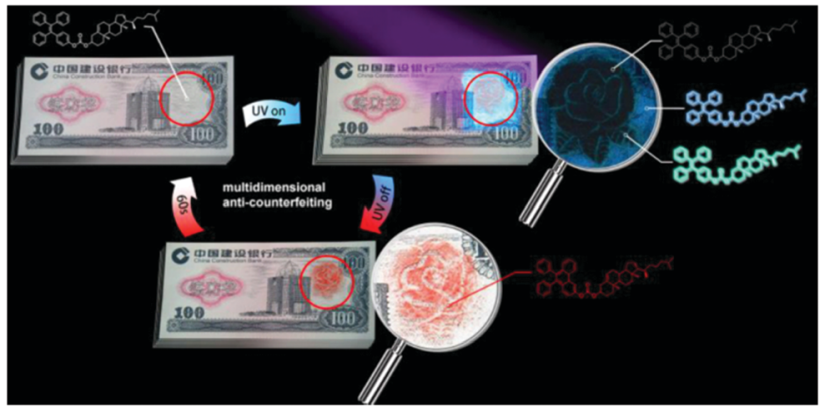

Fig. 37 Illustration of the prototype design based on a single-molecule structure of $\mathbf{4 0}$ in multidimensional anticounterfeiting applications on an example banknote. Reproduced with permission. ${ }^{49}$ Copyright 2019, Wiley-VCH. practical applications. Herein, we have reviewed the recent progresses made in AIE-active photochromic materials under the categories of molecular structures, material morphologies, and practical applications. It is clear that the introduction of the AIE property into photochromic molecules is a direct but efficient strategy to avoid the ACQ problem for designing practical organic fluorescent materials with efficient emissions in their solid or aggregated states. According to the reported molecular structures, four methods have been demonstrated to induce AIE into a photochromic system: (1) directly mixing AIEgens with photochromic molecules; (2) combination of AIEgens and photochromic molecules via chemical bonding; (3) combination of AIEgens and photochromic molecules via host-guest interactions; and (4) new molecular system design. Moreover, efforts on organic NPs, gels, polymers, LCs, and supramolecules have also been made to explore practical applications in data recording and storage, logic gate, super-resolution fluorescent imaging, chemosensor, anticounterfeiting, and other fields. Obviously, investigations on novel AIE-active photochromic systems are still insufficient and their properties are still not sufficiently worthwhile to match the requirements of practical applications. A thorough understanding of the relationships between structures and performances is the key to tune such photochromic systems to afford higher quantum yields, faster switching speeds, better thermal stability, and higher fatigue resistance. Besides, photochromic materials with biological compatibility (visible- or near-infrared-light drive, water solubility, etc.) are becoming a hot topic in biomedicine, such as cell imaging, pharmacological chemotherapy, and drug delivery. Considering the achievements that have been made at present and the challenges that remain, we believe that AIE-active photochromic materials can be developed at a higher speed and utilized in our daily lives. We hope that this review can provide ideas toward molecular design and inspire more researchers to work in this meaningful and challenging area.

\section{Conflicts of interest}

There are no conflicts to declare.

\section{Acknowledgements}

We gratefully acknowledge the financial supported by the National Natural Science Foundation of China (21878136, and 21372194), Guangdong Basic and Applied Research Key Foundation (2018KZDXM045), Young Innovative Talents Project of General Colleges and Universities in Guangdong Province (2018KQNCX149), Special Research Funds from Lingnan Normal University (ZL2014).

\section{Notes and references}

1 J. Teyssier, S. V. Saenko, D. Marel and M. C. Milinkovitch, Photonic crystals cause active colour change in chameleons, Nat. Commun., 2015, 6, 6368. 
2 (a) J. Fritsche, Photochromism of tetracene, C. R. Acad. Sci., 1867, 69, 1035; (b) W. Marckwald, Über phototropie, Z. Phys. Chem., 1899, 30, 140-145; (c) Y. Hirshberg, Reversible formation and eradication of colors by irradiation at low temperatures. A photochemical memory model, J. Am. Chem. Soc., 1956, 78, 2304-2312; (d) M. Irie, Photochromism: memories and switches-introduction, Chem. Rev., 2000, 100, 1683-1684; (e) H. Bouas-Laurent and H. Durr, Organic photochromism (IUPAC technical report), Pure Appl. Chem., 2001, 73, 639-665; $(f)$ B. L. Feringa, Molecular switches, Wiley-VCH, Weinheim, Germany, 2001, vol. 42; ( $g$ ) A. V. E. El'tsov, Organic photochromes, Springer, New York, 1990.

3 (a) P. Bamfield and M. G. Hutchings, Chromic phenomena: technological applications of colour chemistry, RSC Publishing, Cambridge, UK, 2nd edn, 2010; (b) C. Reus and T. Baumgartner, Dalton Trans., 2016, 45, 1850-1855.

4 (a) J. W. Chung, Y. You, H. S. Huh, B. K. An, S. J. Yoon, S. H. Kim, S. W. Lee and S. Y. Park, Shear- and UV-induced fluorescence switching in stilbenic $\pi$-dimer crystals powered by reversible [2+2] cycloaddition, J. Am. Chem. Soc., 2009, 131, 8163-8172; (b) D. A. Davis, A. Hamilton, J. L. Yang, L. D. Cremar, D. Van Gough, S. L. Potisek, M. T. Ong, P. V. Braun, T. J. Martinez, S. R. White, J. S. Moore and N. R. Sottos, Force-induced activation of covalent bonds in mechanoresponsive polymeric materials, Nature, 2009, 459, 68-72; (c) Z. Chi, X. Zhang, B. Xu, X. Zhou, C. Ma, Y. Zhang, S. Liu and J. Xu, Recent advances in organic mechanofluorochromic materials, Chem. Soc. Rev., 2012, 41, 3878-3896; (d) T. Han, Y. N. Hong, N. Xie, S. J. Chen, N. Zhao, E. G. Zhao, J. W. Y. Lam, H. H. Y. Sung, Y. P. Dong, B. Tong and B. Z. Tang, Defect-sensitive crystals based on diaminomaleonitrile-functionalized Schiff base with aggregation-enhanced emission, J. Mater. Chem. C, 2013, 1, 7314-7320; (e) J. Sun, J. Han, Y. Liu, Y. Duan, T. Han and J. Yuan, Mechanochromic luminogen with aggregation-induced emission: implications for ink-free rewritable paper with high fatigue resistance and low toxicity, J. Mater. Chem. C, 2016, 4, 8276-8283.

5 (a) Y. Zimenkov, S. N. Dublin, R. Ni, R. S. Tu, V. Breedveld, R. P. Apkarian and V. P. Conticello, Rational design of a reversible $\mathrm{pH}$-responsive switch for peptide self-assembly, J. Am. Chem. Soc., 2006, 128, 6770-6771; (b) C. RomeroNieto, S. Durben, I. M. Kormos and T. Baumgartner, Simple and efficient generation of white light emission from organophosphorus building blocks, Adv. Funct. Mater., 2009, 19, 3625-3631; (c) M. Stolar and T. Baumgartner, Synthesis and unexpected halochromism of carbazole-functionalized dithienophospholes, New J. Chem., 2012, 36, 1153-1160; (d) H. V. Huynh, X.-M. He and T. Baumgartner, Halochromic generation of white light emission using a single dithienophosphole luminophore, Chem. Commun., 2013, 49, 4899-4901; (e) J. Liu, Y. Huang, A. Kumar, A. Tan, S. Jin, A. Mozhi and X. J. Liang, pH-Sensitive nano-systems for drug delivery in cancer therapy, Biotechnol. Adv., 2014, 32, 693-710.

6 (a) J. C. Crano and R. J. Guglielmetti, Organic photochromic and thermochromic compounds: physicochemical studies, biological applications, and thermochromism, Springer, New York, 1999, vol. 2; (b) Y. Ren, W. H. Kan, M. A. Henderson, P. G. Bomben, C. P. Berlinguette, V. Thangadurai and T. Baumgartner, External-stimuli responsive photophysics and liquid crystal properties of self-assembled "phosphole-lipids", J. Am. Chem. Soc., 2011, 133, 17014-17026; (c) S. Datta and S. Bhattacharya, Evidence of aggregation induced emission enhancement and keto-enol-tautomerism in a gallic acid derived salicylideneaniline gel, Chem. Commun., 2012, 48, 877-879.

7 (a) Y. F. Feng, Y. L. Yan, S. Wang, W. H. Zhu, S. X. Qian and H. Tian, Photochromic thiophene oligomers based on bisthienylethene: syntheses, photochromic and two-photon properties, J. Mater. Chem., 2006, 16, 3685-3692; (b) C. J. Carling, J. C. Boyer and N. R. Branda, Remote-control photoswitching using NIR light, J. Am. Chem. Soc., 2009, 131, 10838-10839; (c) I. Takahashi, Y. Honda and S. Hirota, Regulating copper-binding affinity with photoisomerizable azobenzene ligand by construction of a self-Assembled monolayer, Angew. Chem., Int. Ed., 2009, 48, 6065-6068; (d) P. Zacharias, M. C. Gather, A. Kohnen, N. Rehmann and K. Meerholz, Photoprogrammable organic light-emitting diodes, Angew. Chem., Int. Ed., 2009, 48, 4038-4041; (e) M. Irie, Y. Yokoyama and T. Seki, New frontiers in photochromism, Springer, 2013.

8 (a) H. Durr and H. Bouas-Laurent, Photochromism: molecules and systems, Elsevier B. V., Amsterdam, Netherlands, 2003; (b) S. Hecht, Optical switching of hierarchical self-assembly: towards "enlightened" materials, Small, 2005, 1, 26-29; (c) A. C. Whalley, M. L. Steigerwald, X. Guo and C. Nuckolls, Reversible switching in molecular electronic devices, J. Am. Chem. Soc., 2007, 129, 12590-12591; (d) V. Ferri, M. Elbing, G. Pace, M. D. Dickey, M. Zharnikov, P. Samori, M. Mayor and M. A. Rampi, Light-powered electrical switch based on cargo-lifting azobenzene monolayers, Angew. Chem., Int. Ed., 2008, 47, 3455-3457.

9 (a) S. Yagai and A. Kitamura, Recent advances in photoresponsive supramolecular self-assemblies, Chem. Soc. Rev., 2008, 37, 1520-1529; (b) W. Szymanski, J. M. Beierle, H. A. V. Kistemaker, W. A. Velema and B. L. Feringa, Reversible photocontrol of biological systems by the incorporation of molecular photoswitches, Chem. Rev., 2013, 113, 6114-6178; (c) J. Zhang, J. Wang and H. Tian, Taking orders from light: progress in photochromic bio-materials, Mater. Horiz., 2014, 1, 169-184; (d) D. H. Qu, Q. C. Wang, Q. W. Zhang, X. Ma and H. Tian, Photoresponsive hostguest functional systems, Chem. Rev., 2015, 115, 7543-7588.

10 (a) J. F. Xu, Y. Z. Chen, D. Wu, L. Z. Wu, C. H. Tung and Q. Z. Yang, Photoresponsive hydrogen-bonded supramolecular polymers based on a stiff stilbene unit, Angew. Chem., Int. Ed., 2013, 125, 9920-9924; (b) F. Huang and L. Isaacs, Guest editorial: responsive host-guest systems, Acc. Chem. Res., 2014, 47, 1923-1924; (c) J. Park, D. Feng, S. Yuan and H. C. Zhou, Photochromic metal-organic frameworks: reversible control of singlet oxygen generation, Angew. Chem., Int. Ed., 2015, 127, 440-445; (d) X. Yao, T. Li, J. Wang, X. Ma and H. Tian, Recent Progress in 
Photoswitchable supramolecular self-assembling systems, Adv. Opt. Mater., 2016, 4, 1322-1349.

11 (a) S. Sortino, Photoactivated nanomaterials for biomedical release applications, J. Mater. Chem., 2012, 22, 301-318; (b) A. Y. Rwei, W. Wang and D. S. Kohane, Photoresponsive nanoparticles for drug delivery, Nano Today, 2015, 10, 451-467; (c) J. Olejniczak, C. J. Carling and A. Almutairi, Photocontrolled release using one-photon absorption of visible or NIR light, J. Controlled Release, 2015, 219, 18-30; (d) S. Jia, W. K. Fong, B. Graham and B. J. Boyd, Photoswitchable molecules in long-wavelength light-responsive drug delivery: from molecular design to applications, Chem. Mater., 2018, 30, 2873-2887; (e) K. Hull, J. Morstein and D. Trauner., In vivo photopharmacology, Chem. Rev., 2018, 118, 10710-10747.

12 (a) R. S. Stoll, M. V. Peters, A. Kuhn, S. Heiles, R. Goddard, M. Bühl, C. M. Thiele and S. Hecht, Photoswitchable catalysts: correlating structure and conformational dynamics with reactivity by a combined experimental and computational approach, J. Am. Chem. Soc., 2008, 131, 357-367; (b) T. Imahori, R. Yamaguchi and S. Kurihara, Azobenzene-tethered bis(trityl alcohol) as a photoswitchable cooperative acid catalyst for Morita-Baylis-Hillman reactions, Chem. - Eur. J., 2012, 18, 10802-10807; (c) B. M. Neilson and C. W. Bielawski, Illuminating photoswitchable catalysis, ACS Catal., 2013, 3, 1874-1885; (d) T. Imahori and S. Kurihara, Stimuli-responsive cooperative catalysts based on dynamic conformational changes toward spatiotemporal control of chemical reactions, Chem. Lett., 2014, 43, 1524-1531; (e) S. P. Ihrig and F. Eisenreich, Stefan Hecht. Photoswitchable polymerization catalysis: state of the art, challenges, and perspectives, Chem. Commun., 2019, 55, 4290-4298.

13 (a) D. H. Waldeck, Photoisomerization dynamics of stilbenes, Chem. Rev., 1991, 91, 415-436; (b) N. Tamai and H. Miyasaka, Ultrafast dynamics of photochromic systems, Chem. Rev., 2000, 100, 1875-1890; (c) H. M. Bandara and S. C. Burdette, Photoisomerization in different classes of azobenzene, Chem. Soc. Rev., 2012, 41, 1809-1825; (d) E. Merino, Synthesis of azobenzenes: the coloured pieces of molecular materials, Chem. Soc. Rev., 2011, 40, 3835-3853; (e) M. Han and Y. Norikane, Photoisomerization and light-driven fluorescence enhancement of azobenzene derivatives, Wiley, Chichester, UK, 2014, pp. 185-204; $(f)$ M. Baroncini and G. Bergamini, Azobenzene in molecular and supramolecular devices and machines, Wiley-VCH, Weinheim, Germany, 2014, pp. 379-397.

14 (a) M. Irie and M. Mohri, Thermally irreversible photochromic systems. Reversible photocyclization of diarylethene derivatives, J. Org. Chem., 1988, 53, 803-808; (b) T. He and S. Yang, Recent progresses on diarylethene based photochromic switches, Chem. Soc. Rev., 2004, 35, 85-97; (c) Multifunctional diarylethenes, ed. B. L. Feringa and W. R. Browne, Wiley-VCH, Weinheim, Germany, 2011; (d) M. Irie, T. Fukaminato, K. Matsuda and S. Kobatake, Photochromism of diarylethene molecules and crystals: memories, switches, and actuators, Chem. Rev., 2014, 114, 12174-12277.
15 (a) R. C. Bertelson, Organic photochromic and thermochromic compounds, Kluwer Academic Publishers, New York, 2002, pp. 11-83; (b) L. Florea, D. Diamond and F. Benito-Lopez, Photo-responsive polymeric structures based on spiropyran, Macromol. Mater. Eng., 2012, 297, 1148-1159; (c) R. Klajn, Spiropyran-based dynamic materials, Chem. Soc. Rev., 2014, 43, 148-184; (d) A. Fihey, A. Perrier, W. R. Browne and D. Jacquemin, Multiphotochromic molecular systems, Chem. Soc. Rev., 2015, 44, 3719-3759.

16 (a) E. Hadjoudis and I. M. Mavridis, Photochromism and thermochromism of Schiff bases in the solid state: structural aspects, Chem. Soc. Rev., 2004, 33, 579-588; (b) E. Hadjoudis, S. Chatziefthimiou and I. Mavridis, Anils: Photochromism by H-transfer, Curr. Org. Chem., 2009, 13, 269-286.

17 (a) S. Helmy, F. A. Leibfarth, S. Oh, J. E. Poelma, C. J. Hawker and J. R. d. Alaniz, Photoswitching using visible light: a new class of organic photochromic molecules, J. Am. Chem. Soc., 2014, 136, 8169-8172; (b) S. Helmy, S. Oh, F. A. Leibfarth, C. J. Hawker and J. R. d. Alaniz, Design and synthesis of donor-acceptor stenhouse adducts: a visible light photoswitch derived from furfural, J. Org. Chem., 2014, 79, 11316-11329.

18 (a) A. P. De Silva, N. D. McClenaghan and C. P. McCoy, Molecular Switches, Wiley-VCH, Weinheim, Germany, 2001; (b) M. M. Russew and S. Hecht, Photoswitches: from molecules to materials, Adv. Mater., 2010, 22, 3348-3360; (c) A. V. e. El'tsov, Organic Photochromes, Springer, New York, 2012; (d) J. Zhang, Q. Zou and H. Tian, Photochromic materials: more than meets the eye, Adv. Mater., 2013, 25, 378-399.

19 J. Luo, Z. Xie, J. W. Y. Lam, L. Cheng, H. Chen, C. Qiu, H. S. Kwok, X. Zhan, Y. Liu, D. Zhu and B. Z. Tang, Aggregation-induced emission of 1-methyl-1,2,3,4,5-pentaphenylsilole., Chem. Commun., 2001, 1740-1741.

20 (a) J. Mei, N. L. C. Leung, R. T. K. Kwok, J. W. Y. Lam and B. Z. Tang, Aggregation-induced emission: together we shine, united we soar, Chem. Rev., 2015, 115, 11718-11940; (b) Z. Wang, J. Nie, W. Qin, Q. Hu and B. Z. Tang, Gelation process visualized by aggregation-induced emission fluorogens, Nat. Commun., 2016, 7, 12033; (c) Y. Chen, W. Zhang, Z. Zhao, Y. Cai, J. Gong, R. T. K. Kwok, J. W. Y. Lam, H. H. Y. Sung, I. D. Williams and B. Z. Tang, An easily accessible ionic aggregation-induced emission luminogen with hydrogen-bonding-switchable emission and wash-free imaging ability, Angew. Chem., Int. Ed., 2018, 57, 5011-5109; (d) H. Ding, J. Li, G. Xie, G. Lin, R. Chen, Z. Peng, C. Yang, B. Wang, J. Sun and C. Wang, An AIEgen-based 3D covalent organic framework for white light-emitting diodes, Nat. Commun., 2018, 9, 5234; (e) G. Feng and B. Liu, Aggregationinduced emission (AIE) dots: emerging theranostic nanolights, Acc. Chem. Res., 2018, 51, 1404-1414; $(f)$ X. Ni, X. Zhang, X. Duan, H. L. Zheng, X. S. Xue and D. Ding, Near-infrared afterglow luminescent aggregation-induced emission dots with ultrahigh tumor-to-liver signal ratio for promoted image-guided cancer surgery, Nano Lett., 2019, 19, 318-330.

21 (a) N. H. Xie, Y. Chen, H. Ye, C. Li and M. Q. Zhu, Progress on photochromic diarylethenes with aggregation induced 
emission, Front. Optoelectron., 2018, 11, 317-332; (b) J. Mei, Y. Hong, J. W. Y. Lam, A. Qin, Y. Tang and B. Z. Tang, Aggregation-induced emission: the whole is more brilliant than the parts, Adv. Mater., 2014, 26, 5429-5479.

22 H. Dong, M. Luo, S. Wang and X. Ma, Synthesis and properties of tetraphenylethylene derivatived diarylethene with photochromism and aggregation-induced emission, Dyes Pigm., 2017, 139, 118-128.

23 L. Ma, C. Li, Q. Yan, S. Wang, W. Miao and D. Cao, Unsymmetrical photochromic bithienylethene-bridge tetraphenylethene molecular switches: synthesis, aggregationinduced emission and information storage, Chin. Chem. Lett., 2020, 31, 361-364.

24 S. Chen, W. Li, X. Li and W. H. Zhu, Aggregation-controlled photochromism based on a dithienylethene derivative with aggregation-induced emission, J. Mater. Chem. C, 2017, 5, 2717-2722.

25 L. Kono, Y. Nakagawa, A. Fujimoto, R. Nishimura, Y. Hattori, T. Mutai, N. Yasuda, K. Koizumi, S. Yokojima, S. Nakamura and K. Uchida, Aggregation-induced emission effect on turn-off fluorescent switching of a photochromic diarylethene, Beilstein J. Org. Chem., 2019, 15, 2204-2212.

26 Q. Luo, F. Cao, C. Xiong, Q. Dou and D. H. Qu, Hybrid cis/ trans tetraarylethenes with switchable aggregation-induced emission (AIE) and reversible photochromism in the solution, PMMA film, solid powder, and single crystal, J. Org. Chem., 2017, 82, 10960-10967.

27 Q. Qi, J. Qian, S. Ma, B. Xu, S. X. A. Zhang and W. Tian, Reversible multistimuli-response fluorescent switch based on tetraphenylethene-spiropyran molecules, Chem. - Eur. J., 2014, 20, 1-8.

28 Q. Yu, X. Su, T. Zhang, Y. M. Zhang, M. Li, Y. Liu and S. X. A. Zhang, Non-invasive fluorescence switch in polymer films based on spiropyran-photoacid modified TPE, J. Mater. Chem. C, 2018, 6, 2113-2122.

29 T. Lin, Xing Su, K. Wang, M. Li, H. Guo, L. Liu, B. Zou, Y. M. Zhang, Y. Liu and S. X. A. Zhang, An AIE fluorescent switch with multi-stimuli responsive property and application for quantitatively detecting $\mathrm{pH}$ value, sulfite anion and hydrostatic pressure, Mater. Chem. Front., 2019, 3, 1052-1061.

30 Q. Qi, Chong Li, X. Liu, S. Jiang, Z. Xu, R. Lee, M. Zhu and $\mathrm{Bin} \mathrm{Xu}$ and $\mathrm{W}$. Tian, Solid-state photoinduced luminescence switch for advanced anticounterfeiting and super-resolution imaging applications, J. Am. Chem. Soc., 2017, 139, 16036-16039.

31 S. Peng, J. Wen, M. Hai, Z. Yang, X. Yuan, D. Wang, H. Cao and W. He, Synthesis and application of reversible fluorescent photochromic molecules based on tetraphenylethylene and photochromic groups, New J. Chem., 2019, 43, 617-621.

32 X. Li, C. Li, S. Wang, H. Dong, X. Ma and D. Cao, Synthesis and properties of photochromic spirooxazine with aggregationinduced emission fluorophores polymeric nanoparticles, Dyes Pigm., 2017, 142, 481-490.

33 H. M. D. Bandara and S. C. Burdette, Photoisomerization in different classes of azobenzene, Chem. Soc. Rev., 2012, 41, 1809-1825.
34 B. K. Tsai, C. H. Chen, C. H. Hung, V. K. S. Hsiao and C. C. Chu, Photoswitchable fluorescence on/off behavior between cis- and trans-rich azobenzenes, J. Mater. Chem., 2012, 22, 20874-20877.

35 M. Luo, S. Wang, M. Wang, S. Huang, C. Li, L. Chen and $\mathrm{X}$. Ma, Novel organogel harnessing Excited-State Intramolecular Proton Transfer process with aggregation induced emission and photochromism, Dyes Pigm., 2016, 132, 48-57.

36 L. Wang, Y. Li, X. You, K. Xu, Q. Feng, J. Wang, Y. Liu, K. Li and $\mathrm{H}$. Hou, Erasable photo-patterning material based on a specially designed 4-(1,2,2-triphenylvinyl)aniline salicylaldehyde hydrazone aggregation-induced emission (AIE) molecule, J. Mater. Chem. C, 2017, 5, 65-72.

37 H. Sun, J. Y. Li, F. F. Han, R. Zhang, Y. Zhao, B. X. Miao and Z. H. Ni, Reversible photochromic tetraphenylethene-based Schiff base: design, synthesis, crystal structure and applications as visible light driven rewritable paper and UV sensor, Dyes Pigm., 2019, 167, 143-150.

38 T. Ning, L. Liu, D. Jia, X. Xie and D. Wu, Aggregationinduced emission, photochromism and self-assembly of pyrazolone phenlysemicarbazones, J. Photochem. Photobiol., A, 2014, 291, 48-53.

39 J. Chai, Y. Wu, B. Yang and B. Liu, The photochromism, light harvesting and self-assembly activity of a multifunction Schiff-base compound based on the AIE effect, J. Mater. Chem. C, 2018, 6, 4057-4064.

40 L. Peng, Y. Zheng, X. Wang, A. Tong and Y. Xiang, Photoactivatable aggregation-induced emission fluorophores with multiple-color fluorescence and wavelength-selective activation, Chem. - Eur. J., 2015, 21, 4326-4332.

41 (a) B. Wu, T. Xue, W. Wang, S. Li, J. Shen and Y. He, Visible Light Triggered Aggregation-induced Emission Switching with Donor-acceptor Stenhouse Adduct, J. Mater. Chem. C, 2018, 6, 8538-8545; (b) Q. Yan, C. Li, S. Wang, Z. Lin, Q. Yan and D. Cao, Dyes Pigm., 2020, 178, 108352.

42 P. Wei, J. X. Zhang, Z. Zhao, Y. Chen, X. He, M. Chen, J. Gong, H. H. Y. Sung, I. D. Williams, J. W. Y. Lam and B. Z. Tang, Multiple yet controllable photoswitching in a single AIEgen system, J. Am. Chem. Soc., 2018, 140, 1966-1975.

43 X. Mei, J. Wang, Z. Zhou, S. Wu, L. Huang, Z. Lin and Q. Ling, Diarylmaleic anhydrides: unusual organic luminescence, multi-stimuli response and photochromism, J. Mater. Chem. C, 2017, 5, 2135-2141.

44 L. Huang, Y. Qiu, C. Wu, Z. Ma, Z. Shen and X. Jia, A multistate fluorescent switch with multifunction of AIE, methanol responsiveness, photochromism and mechanochromism, J. Mater. Chem. C, 2018, 6, 10250-10255.

45 (a) Z. He, L. Shan, J. Mei, H. Wang, J. W. Y. Lam, H. H. Y. Sung, I. D. Williams, X. Gu, Q. Miao and B. Z. Tang, Aggregation-induced emission and aggregation promoted photochromism of bis(diphenylmethylene)dihydroacenes, Chem. Sci., 2015, 6, 3538-3543; (b) Z. Zhou, S. Xie, X. Chen, Y. Tu, J. Xiang, J. Wang, Z. He, Z. Zeng and B. Z. Tang, Spirofunctionalized diphenylethenes: suppression of a reversible photocyclization contributes to the aggregation-induced emission effect, J. Am. Chem. Soc., 2019, 141, 9803-9807. 
46 D. Ou, T. Yu, Z. Yang, T. Luan, Z. Mao, Y. Zhang, S. Liu, J. Xu, Z. Chi and M. R. Bryce, Combined aggregation induced emission (AIE), photochromism and photoresponsive wettability in simple dichloro-substituted triphenylethylene derivatives, Chem. Sci., 2016, 7, 5302-5306.

47 T. Yu, D. Ou, L. Wang, S. Zheng, Z. Yang, Y. Zhang, Z. Chi, S. Liu, J. Xu and M. P. Aldred, A new approach to switchable photochromic materials by combining photochromism and piezochromism together in an AIE-active molecule, Mater. Chem. Front., 2017, 1, 1900-1904.

48 L. Wang, T. Yu, Z. Xie, E. Ubba, T. Zhan, Z. Yang, Y. Zhang and Z. Chi, Gated photochromic molecules with AIEgen: turnon the photochromism with an oxidation reagent, $R S C$ Adv., 2018, 8, 18613-18618.

49 G. Huang, Q. Xia, W. Huang, J. Tian, Z. He, B. S. Li and B. Z. Tang, Multiple Anti-counterfeiting Guarantees from a Simple Tetraphenylethylene Derivative - High-contrasted and Multi-state Mechanochromism and Photochormism, Angew. Chem., Int. Ed., 2019, 131, 17978-17983.

50 (a) D. J. Abdallah and R. G. Weiss, Organogels and low molecular mass organic gelators, Adv. Mater., 2000, 12, 1237-1247; (b) M. S. Neralagatta and M. Uday, Supramolecular gels: functions and uses, Chem. Soc. Rev., 2005, 34, 821-836; (c) R. G. Weiss, The past, present, and future of molecular gels. What is the status of the field, and where is it going?, J. Am. Chem. Soc., 2014, 136, 7519-7530; (d) S. S. Babu, V. K. Praveen and A. Ajayaghosh, Functional $\pi$-gelators and their applications, Chem. Rev., 2014, 114, 1973-2129.

51 J. W. Chung, S. J. Yoon, S. J. Lim, B. K. An and S. Y. Park, Dual-mode switching in highly fluorescent organogels: binary logic gates with optical/thermal inputs, Angew. Chem., Int. Ed., 2009, 48, 7030-7034.

52 D. Kim, J. E. Kwon and S. Y. Park, Fully reversible multistate fluorescence switching: organogel system consisting of luminescent cyanostilbene and turn-on diarylethene, Adv. Funct. Mater., 2018, 28, 1706213.

53 Q. Chen, D. Zhang, G. Zhang, X. Yang, Y. Feng, Q. Fan and D. Zhu, Multicolor tunable emission from organogels containing tetraphenylethene, perylenediimide, and spiropyran derivatives, Adv. Funct. Mater., 2010, 20, 3244-3251.

54 L. Shi, X. Ran, Y. Li, Q. Li, W. Qiu and L. Guo, Photoresponsive structure transformation and emission enhancement based on a tapered azobenzene gelator, RSC Adv., 2015, 5, 38283-38289.

55 (a) Q. Li, Liquid crystals beyond displays: Chemistry, physics, and applications, John Wiley \& Sons, Inc., Hoboken, NJ, 2012; (b) R. J. Bushby, S. M. Kelly and M. O'Neill, Liquid crystalline semiconductors: Materials, properties and applications, Springer, Dordrecht, 2013; (c) Q. Li, Nanoscience with liquid crystals: from self-organized nanostructures to applications, Springer-Verlag, Heidelberg, 2014.

56 (a) S. J. Woltman, G. D. Jay and G. P. Crawford, Liquid crystals: frontiers in biomedical applications, World Scientific, New Jersey, USA, 2007; (b) R. H. Chen, Liquid crystal displays: Fundamental physics and technology, John Wiley \& Sons, Inc.,
Hoboken, NJ, 2011; (c) J. W. Goodby, P. J. Collings, T. Kato, C. Tschierske, H. Glesson and P. Raynes, Handbook of liquid crystals, Wiley-VCH Verlag GmbH, Weinheim, 2nd edn, 2014.

57 (a) K. Ichimura, Photoalignment of liquid-crystal systems, Chem. Rev., 2000, 100, 1847-1873; (b) G. Berkovic, V. Krongauz and V. Weiss, Spiropyrans and spirooxazines for memories and switches, Chem. Rev., 2000, 100, 1741-1753; (c) Y. Wang and Q. Li, Light-driven chiral molecular switches or motors in liquid crystals, Adv. Mater., 2012, 24, 1926-1945.

58 X. Yu, H. Chen, X. Shi, P. A. Albouy, J. Guo, J. Hu and M. H. Li, Liquid crystal gelators with photo-responsive and AIE properties, Mater. Chem. Front., 2018, 2, 2245-2253.

59 S. J. Lim, B. K. An and S. Y. Park, Bistable photoswitching in the film of fluorescent photochromic polymer: enhanced fluorescence emission and its high contrast switching, Macromolecules, 2005, 38, 6236-6239.

60 G. Sinawang, J. Wang, B. Wu, X. Wang and Y. He, Photoswitchable aggregation-induced emission polymer containing dithienylethene and tetraphenylethene moieties, RSC Adv., 2016, 6, 12647-12651.

61 R. Singh, H. Y. Wu, A. K. Dwivedi, A. Singh, C. M. Lin, P. Raghunath, M. C. Lin, T. K. Wu, K. H. Wei and H. C. Lin, Monomeric and aggregation emissions of tetraphenylethene in a photo-switchable polymer controlled by cyclization of diarylethene and solvent conditions, J. Mater. Chem. C, 2017, 5, 9952-9962.

62 C. Xiong, L. Wang, W. Li and Q. Luo, Photochromic monomer and coordination polymer based hybrid tetra-arylethenes, Chem. Lett., 2018, 47, 1127-1130.

63 Y. Li, N. Zhou, W. Zhang, F. Zhang, J. Zhu, Z. Zhang, Z. Cheng, Y. Tu and X. Zhu, Light-driven and aggregationinduced emission from side-chain azoindazole polymers, J. Polym. Sci., Part A: Polym. Chem., 2011, 49, 4911-4920.

64 B. Wu, W. Wang, J. Wang, S. Li and Y. He, Redox triggered aggregation induced emission (AIE) polymers with azobenzene pendants, Dyes Pigm., 2018, 157, 290-297.

65 P. Q. Nhien, W. L. Chou, T. T. K. Cuc, T. M. Khang, C. H. Wu, N. Thirumalaivasan, B. T. B. Hue, J. I. Wu, S. P. $\mathrm{Wu}$ and H. C. Lin, Multi-stimuli responsive FRET processes of bifluorophoric AIEgens in an amphiphilic copolymer and its application to cyanide detection in aqueous media, ACS Appl. Mater. Interfaces, 2020, 12, 10959-10972.

66 S. J. Lim, B. K. An, S. D. Jung, M. A. Chung and S. Y. Park, Photoswitchable organic nanoparticles and a polymer film employing multifunctional molecules with enhanced fluorescence emission and bistable photochromism, Angew. Chem., Int. Ed., 2004, 43, 6346-6350.

67 (a) S. Wang, F. Wang, C. Li, T. Li, D. Cao and X. Ma, Photo-induced morphology transition of a multifunctional photochromic bisthienylethene molecule with switchable aggregation-induced emission, Sci. China: Chem., 2018, 61, 1301-1306; (b) M. Q. Zhu, Both self-assembly and aggregationinduced emission are photoswitchable, Sci. China: Chem., 2018, 61, 1201-1202. 
68 C. Li, W. L. Gong, Z. Hu, M. P. Aldred, G. F. Zhang, T. Chen, Z. L. Huang and M. Q. Zhu, Photoswitchable aggregationinduced emission of a dithienylethene-tetraphenylethene conjugate for optical memory and super-resolution imaging, RSC Adv., 2013, 3, 8967-8972.

69 J. Qi, C. Chen, X. Zhang, X. Hu, S. Ji, R. T. K. Kwok, J. W. Y. Lam, D. Ding and B. Z. Tang, Light-driven transformable optical agent with adaptive functions for boosting cancer surgery outcomes, Nat. Commun., 2018, 9, 1848.

70 X. Sheng and Y. Qian, Photoswitchable composite organic nanoparticles with aggregation-induced enhanced emission, J. Nanosci. Nanotechnol., 2010, 10, 8307-8311.

71 L. Chen, J. Zhang, Q. Wang and L. Zou, Photo-controllable and aggregation-induced emission based on photochromic bithienylethene, Dyes Pigm., 2015, 123, 112-115.

72 W. Zhong, X. Zeng, J. Chen, Y. Hong, L. Xiao and P. Zhang, Photoswitchable fluorescent polymeric nanoparticles for rewritable fluorescence patterning and intracellular dualcolor imaging with AIE-based fluorogens as FRET donors, Polym. Chem., 2017, 8, 4849-4855.

$73 \mathrm{~J}$. Ji, X. Li, T. Wu and F. Feng, Spiropyran in nanoassemblies as a photosensitizer for photoswitchable ROS generation in living cells, Chem. Sci., 2018, 9, 5816-5821.

74 R. Dong, S. P. Ravinathan, L. Xue, N. Li, Y. Zhang, L. Zhou, C. Cao and X. Zhu, Dual-responsive aggregation-induced emission-active supramolecular nanoparticles for gene delivery and bioimaging, Chem. Commun., 2016, 52, 7950-7953.

75 (a) Y. Hong, J. W. Y. Lam and B. Z. Tang, Aggregationinduced emission, Chem. Soc. Rev., 2011, 40, 5361-5388; (b) D. Ding, K. Li, B. Liu and B. Z. Tang, Bioprobes based on AIE fluorogens, Acc. Chem. Res., 2013, 46, 2441-2453; (c) R. T. K. Kwok, C. W. T. Leung, J. W. Y. Lam and B. Z. Tang, Biosensing by luminogens with aggregationinduced emission characteristics, Chem. Soc. Rev., 2015, 44, 4228-4238.

76 L. Ma, S. Wang, C. Li, D. Cao, T. Li and X. Ma, Photocontrolled fluorescence on/off switching of a pseudo[3]rotaxane between an AIE-active pillar[5] arene host and a photochromic bithienylethene guest, Chem. Commun., 2018, 54, 2405-2408.

77 S. Bhattacharyya, A. Chowdhury, R. Saha and P. S. Mukherjee, Multifunctional self-assembled macrocycles with enhanced emission and reversible photochromic behaviour, Inorg. Chem., 2019, 58, 3968-3981.

78 L. Wang, Z. Sun, M. Ye, Y. Shao, L. Fang and X. Liu, Fabrication of a cross-linked supramolecular polymer on the basis of cucurbit[8]uril-based host-guest recognition with tunable AIE behaviors, Polym. Chem., 2016, 7, 3669-3673.

79 (a) A. Bobrovsky, N. Boiko and V. Shibaev, A new type of multifunctional material based on dual photochromism of ternary chiral photochromic liquid crystalline copolymers for optical data recording and storage, J. Mater. Chem., 2000, 10, 1075-1081; (b) A. Y. Bobrovsky, N. I. Boiko, V. P. Shibaev and J. H. Wendorff, Cholesteric mixtures with photochemically tunable, circularly polarized fluorescence, Adv. Mater., 2003, 15, 282-287; (c) S. J. Lim, B. K. An, S. D. Jung,
M. A. Chung and S. Y. Park, Photoswitchable organic nanoparticles and a polymer film employing multifunctional molecules with enhanced fluorescence emission and bistable photochromism, Angew. Chem., Int. Ed., 2004, 43, 6346-6350.

80 (a) A. Y. Bobrovsky, N. I. Boiko, V. P. Shibaev, M. A. Kalik and M. M. Krayushkin, Mixture of cholesteric copolymer with dithienylethene photochromic dopant: a new material combining optical properties of cholesterics with photochromism, J. Mater. Chem., 2001, 11, 2004-2007; (b) A. Bobrovsky and V. Shibaev, Immiscible blend of cholesteric copolymers as a new type of material with photoregulated optical properties, J. Mater. Chem., 2002, 12, 1284-1287.

81 G. Liu, Y. M. Zhang, L. Zhang, C. Wang and Y. Liu, Controlled photoerasable fluorescent behaviors with dithienylethenebased molecular turnstile, ACS Appl. Mater. Interfaces, 2018, 10, 12135-12140.

82 (a) A. P. De Silva, H. Q. N. Gunaratne and C. P. Mccoy, A molecular photoionic AND gate based on fluorescent signalling, Nature, 1993, 364, 42-44; (b) M. Frigoli and G. H. Mehl, Multiple addressing in a hybrid biphotochromic system, Angew. Chem., Int. Ed., 2005, 44, 5048-5052; (c) J. Andreasson, U. Pischel, S. D. Straight, T. A. Moore, A. L. Moore and D. Gust, All-photonic multifunctional molecular logic device, J. Am. Chem. Soc., 2011, 133, 11641-11648; (d) Q. Zou, X. Li, J. Zhang, J. Zhou, B. Sun and H. Tian, Unsymmetrical diarylethenes as molecular keypad locks with tunable photochromism and fluorescence via $\mathrm{Cu}^{2+}$ and CN-coordinations, Chem. Commun., 2012, 48, 2095-2097; (e) Y. Wu, Y. Xie, Q. Zhang, H. Tian, W. Zhu and A. D. Li, Quantitative photoswitching in bis (dithiazole) ethene enables modulation of light for encoding optical signals, Angew. Chem., Int. Ed., 2014, 53, 2122-2126.

83 (a) M. Bates, B. Huang, G. T. Dempsey and X. Zhuang, Multicolor super-resolution imaging with photo-switchable fluorescent probes, Science, 2007, 317, 1749; (b) S. J. Lord, N. R. Conley, H. D. Lee, R. Samuel, N. Liu, R. J. Twieg and W. E. Moerner, A photoactivatable push-pull fluorophore for single-molecule imaging in live cells, J. Am. Chem. Soc., 2008, 130, 9204-9205; (c) D. M. Shcherbakova, P. Sengupta, J. Lippincott-Schwartz and V. V. Verkhusha, Photocontrollable fluorescent proteins for superresolution imaging, Annu. Rev. Biophys., 2014, 43, 303-329.

84 (a) J. Zhou, G. Yu and F. Huang, AIE opens new application in super-resolution imaging, J. Mater. Chem. B, 2016, 4, 7761-7765; (b) X. Gu, E. Zhao, T. Zhao, M. Kang, C. Gui, J. W. Y. Lam, S. Du, M. M. T. Loy and B. Z. Tang, A mitochondrion-specific photoactivatable fluorescence turn-on AIE-based bioprobe for localization superresolution microscope, Adv. Mater., 2016, 28, 5064-5071; (c) Y. L. Wang, C. Fan, B. Xin, J. P. Zhang, T. Luo, Z. Q. Chen, Q. Yuan Zhou, Q. Yu, X. N. Li, Z. L. Huang, C. Li, M. Q. Zhu and B. Z. Tang, AIE-based super-resolution imaging probes for $\beta$-amyloid plaques in mouse brain, Mater. Chem. Front., 2018, 2, 1554-1562; (d) Q. Y. Zhou, C. Fan, C. Li, Y. L. Wang, Z. Q. Chen, Q. Yu and M. Q. Zhu, AIE-based universal 
super-resolution imaging for inorganic and organic nanostructures, Mater. Horiz., 2018, 5, 474-479.

85 H. Yang, M. Li, C. Li, Q. Luo, M. Q. Zhu, H. Tian and W. H. Zhu, Unraveling dual aggregation-induced emission behavior in steric hindrance photochromic system for super resolution imaging, Angew. Chem., Int. Ed., 2019, 131, 2-13.

86 (a) M. Natali, L. Soldi and S. Giordani, A photoswitchable Zn(II) selective spiropyran-based sensor, Tetrahedron, 2010, 66, 7612-7617; (b) I. S. Park, Y. S. Jung, K. J. Lee and J. M. Kim, Photoswitching and sensor applications of a spiropyran-polythiophene conjugate, Chem. Commun., 2010, 46, 2859-2861; (c) X. Xie, G. Mistlberger and E. Bakker, Reversible photodynamic chloride-selective sensor based on photochromic spiropyran, J. Am. Chem. Soc., 2012, 134, 16929-16932; (d) S. Heng, A. M. Mak, R. Kostecki, X. Zhang, J. Pei, D. B. Stubing, H. Ebendorff-Heidepriem and A. D. Abell, Photoswitchable calcium sensor: on-off sensing in cells or with microstructured optical fibers, Sens. Actuators, B, 2017, 252, 965-972.

87 (a) H. B. Cheng, Z. Li, Y. D. Huang, L. Liu and H. C. Wu, Pillararene based aggregation-induced-emission-active supramolecular system for simultaneous detection and removal of mercury(II) in water, ACS Appl. Mater. Interfaces, 2017, 9,
11889-11894; (b) D. D. La, S. V. Bhosale, L. A. Jones and S. V. Bhosale, Tetraphenylethylene-based AIE-active probes for sensing applications, ACS Appl. Mater. Interfaces, 2018, 10, 12189-12216.

88 Y. Hong, P. Zhang, H. Wang, M. Yu, Y. Gao and J. Chen, Photoswitchable AIE nanoprobe for lysosomal hydrogen sulfide detection and reversible dual-color imaging, Sens. Actuators, B, 2018, 272, 340-343.

89 (a) J. Kai, L. Zhang, J. Lu, C. Xu, C. Cai and H. Lin, Triplemode emission of carbon dots: applications for advanced anti-counterfeiting, Angew. Chem., 2016, 55, 1-6; (b) W. Tian, J. Zhang, J. Yu, J. Wu, J. Zhang, J. He and F. Wang, Phototunable Full-Color Emission of CelluloseBased Dynamic Fluorescent Materials, Adv. Funct. Mater., 2018, 28, 1703548; (c) C. Shi, X. Shen, Y. Zhu, X. Li, Z. Pang and M. Ge, Excitation wavelength-dependent dual-mode luminescence emission for dynamic multicolor anticounterfeiting, ACS Appl. Mater. Interfaces, 2019, 11, 18548-18554; (d) C. Yang, L. Gu, C. Ma, M. Gu, X. Xie, H. Shi, H. Ma, W. Yao, Z. An and W. Huang, Controllable co-assembly of organic micro/nano heterostructures from fluorescent and phosphorescent molecules for dual anticounterfeiting, Mater. Horiz., 2019, 6, 984-989. 